\title{
Adaptive Coding and Modulation for Large-Scale Antenna Array Based Aeronautical Communications in the Presence of Co-channel Interference
}

\author{
Jiankang Zhang, Member, IEEE, Sheng Chen, Fellow, IEEE, Robert G. Maunder, Senior Member, IEEE, \\ Rong Zhang, Senior Member, IEEE, and Lajos Hanzo, Fellow, IEEE
}

\begin{abstract}
In order to meet the demands of 'Internet above the clouds', we propose a multiple-antenna aided adaptive coding and modulation (ACM) for aeronautical communications. The proposed ACM scheme switches its coding and modulation mode according to the distance between the communicating aircraft, which is readily available with the aid of the airborne radar or the global positioning system. We derive an asymptotic closed-form expression of the signal-to-interference-plus-noise ratio (SINR) as the number of transmitting antennas tends to infinity, in the presence of realistic co-channel interference and channel estimation errors. The achievable transmission rates and the corresponding mode-switching distance-thresholds are readily obtained based on this closed-form SINR formula. Monte-Carlo simulation results are used to validate our theoretical analysis. For the specific example of 32 transmit antennas and 4 receive antennas communicating at a $5 \mathbf{~ G H z}$ carrier frequency and using $6 \mathrm{MHz}$ bandwidth, which are reused by multiple other pairs of communicating aircraft, the proposed distance-based ACM is capable of providing as high as $65.928 \mathrm{Mbps}$ data rate when the communication distance is less than $25 \mathrm{~km}$.
\end{abstract}

Index Terms-Aeronautical communication, large-scale antenna array, Rician channel, adaptive coding and modulation, precoding

\section{INTRODUCTION}

The appealing service of the 'Internet above the clouds' [1] motivates researchers to develop high data rate and high spectral-efficiency (SE) aeronautical communication techniques. Traditionally, satellite-based access has been the main solution for aeronautical communication. However, it suffers from the drawbacks of low throughput and high processing delay as well as high charges by the satellite providers. The aeronautical ad hoc network (AANET) [2] concept was conceived for supporting direct communication and data relaying among aircraft for airborne Internet access. However, the current existing transmission techniques are incapable of providing the high throughput and high SE communications among aircraft required by this airborne Internet access application.

The planed future aeronautical communication systems, specifically, the L-band digital aeronautical communications system (L-DACS) [3], [4] and the aeronautical mobile airport

The authors are with Electronics and Computer Science, University of Southampton, U.K. (E-mails: jz09v@ecs.soton.ac.uk, sqc@ecs.soton.ac.uk, rm@ecs.soton.ac.uk, rz@ecs.soton.ac.uk, lh@ecs.soton.ac.uk).J. Zhang is also with School of Information Engineering, Zhengzhou University, China. S. Chen is also with King Abdulaziz University, Jeddah, Saudi Arabia.

The financial support of the European Research Council's Advanced Fellow Grant, the Royal Society Wolfson Research Merit Award, and the Innovate UK funded Harmonised Antennas, Radios, and Networks (HARNet) are gratefully acknowledged. communication system (AeroMACS) [5], [6], only provide upto $1.37 \mathrm{Mbps}$ and $9.2 \mathrm{Mbps}$ air-to-ground communication date rates, respectively. Moreover, the L-DACS1 air-to-air mode [7] is only capable of providing $273 \mathrm{kbps}$ net user rate for direct aircraft-to-aircraft communication, which cannot meet the high throughput demand of the Internet above the clouds. Furthermore, these rates are achievable for pointto-point transmissions, but multiple frequency resources are required for supporting multiple pairs of aircraft communications. Therefore, these future aeronautical communication techniques fail to satisfy the demanding requirements of airborne Internet access. Additionally, the L-DACS1 air-to-air mode has to collect and distribute the associated channel state information (CSI) to all aircraft within the communication range [7], which is challenging in practical implementation. Even if the air-to-air communication capacity of these future aeronautical communication systems could be made sufficiently high, they would still be forbidden for commercial airborne Internet access, because their frequency bands are within the bands assigned to the safety-critical air traffic control and management systems.

In order to meet the high throughput and high SE demands of the future AANET, we propose a large-scale antenna array aided adaptive coding and modulation (ACM) based solution for aeronautical communication. Before reviewing the family of ACM and multiple-antenna techniques, we first elaborate on the specific choice of the frequency band suitable for the envisaged AANET. Existing air traffic systems mainly use the very high frequency (VHF) band spanning from $118 \mathrm{MHz}$ to $137 \mathrm{MHz}$ [8], and there are no substantial idle frequency bands. The ultra high frequency (UHF) band has almost been fully occupied by television broadcasting, cell phones and satellite communications, including the global positioning system (GPS). Thus, no substantial idle frequency bands can be found in the UHF band either. This motivates us to explore the super high frequency (SHF) band spanning from $3 \mathrm{GHz}$ to $30 \mathrm{GHz}$, for example, using $5 \mathrm{GHz}$ carrier frequency for this aeronautical communication application. Note that even if there were sufficient unused frequency slots in the VHF and UHF bands, it is advisable not to use them because the frequency band of the envisaged airborne Internet access system should be sufficiently far away from the bands assigned to the safety-critical air control and management systems, satellite communication and GPS systems.

ACM [9], [10] has been demonstrated to be a powerful technique of increasing data rate and improving SE over 
wireless fading channels. It has been extensively investigated also in the context of IEEE 802.11 [11], LTE-advance 4G mobile systems [12], [13] and broadband satellite communication systems [14]. The optimal ACM relies on the perfect knowledge of the instantaneous CSI, but channel estimation errors are unavoidable in practical communication systems [15]. Furthermore, the CSI of frequency division duplexing based systems must be obtained through a feedback channel, which potentially introduces feedback errors and delays [16]. These factors significantly degrade the ACM performance. In order to reduce the sensitivity to CSI errors, Zhou et al. [15] proposed an adaptive modulation scheme relying on partial CSI, while Taki et al. [17] designed an ACM scheme based directly on imperfect CSI. A whole range of differentially encoded and non-coherently detected star-QAM schemes were characterized in [18], while the channel coding aspects were documented in [10]. Most existing research on ACM focused on terrestrial wireless communications, where the channels exhibit Rayleigh fading characteristics. But the research community seldom considered the propagation characteristics of aeronautical communications in designing ACM schemes.

In aeronautical communication, typically there is line-ofsight (LOS) propagation, in addition to multipath fading, where the LOS component dominates the other multipath components of the channel. The investigations of [19], [20] have revealed that the aeronautical channel can be modeled as a Rician channel for the flight phases of taxiing, landing, takeoff and en-route, while the aeronautical channel during the aircraft's parking phase can be modeled as a Rayleigh channel, which can be viewed as a specific case of the Rician channel with a zero Rician $K$-factor. Furthermore, multipleantenna aided techniques have been employed in aeronautical communication for increasing the transmission capacity [21]. Although it is challenging to deploy multiple antennas, on an aircraft [22], especially a large-scale antenna array, the development of conformal antenna [23] has paved the way for deploying large-scale antenna arrays on aircraft. At the time of writing, however, there is a paucity of information on how much capacity can be offered by employing multiple antennas in aeronautical communications.

Against this background, we develop an ACM based and

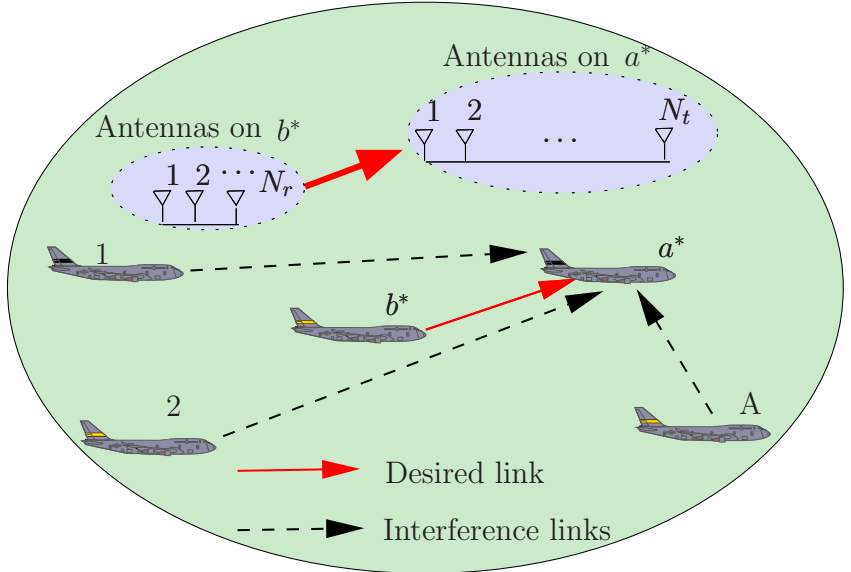

(a) Pilot training $\left(b^{*}\right.$ to $\left.a^{*}\right)$ large-scale antenna array aided physical-layer transmission technique capable of facilitating en-route airborne Internet access for the future AANET employing the time division duplexing (TDD) protocol, which has already been adopted by the so-called automatic dependent surveillance-broadcast standard [24], as well as by the L-DACS and AeroMACS arrangements. Our main contributions are summarized.

1) We propose and analyze a distance-information based ACM scheme for large-scale antenna array aided aeronautical communication in SHF band, which switches its ACM mode based on the distance between the desired pair of communicating aircraft. This scheme is more practical than the existing ACM schemes that rely on instantaneous channel-quality metrics, such as the instantaneous signal-to-noise ratio (SNR). This is because in aeronautical communication it is extremely difficult to acquire an accurate estimate for any instantaneous channel-quality metric, and an ACM based on such a switching metric will frequently fail. By contrast, the accurate distance information between the communicating aircraft can readily be acquired with the aid airborne radar. Alternatively, the accurate position information can also be acquired with the assistance of GPS.

2) We explicitly derive a closed-form expression of the asymptotic signal-to-interference-plus-noise ratio (SINR) of multiple-antenna aided aeronautical communication in the presence of realistic channel estimation errors and the co-channel interference imposed by other aircraft within the communication range. This closed-form SINR formula enables us to directly derive the achievable theoretical transmission rates and the associated mode-switching distance-thresholds for the proposed ACM scheme. Moreover, as a benefit of large-scale antenna arrays, every pair of communicating aircraft in our system uses the same frequency resource block, which dramatically enhances the system's SE.

The rest of this paper is organized as follows. Section II describes the multiple-antenna aided aeronautical communication system, with emphasis on the propagation and signal models. Section III is devoted to the proposed distance-based ACM scheme, including the derivation of the closed-form

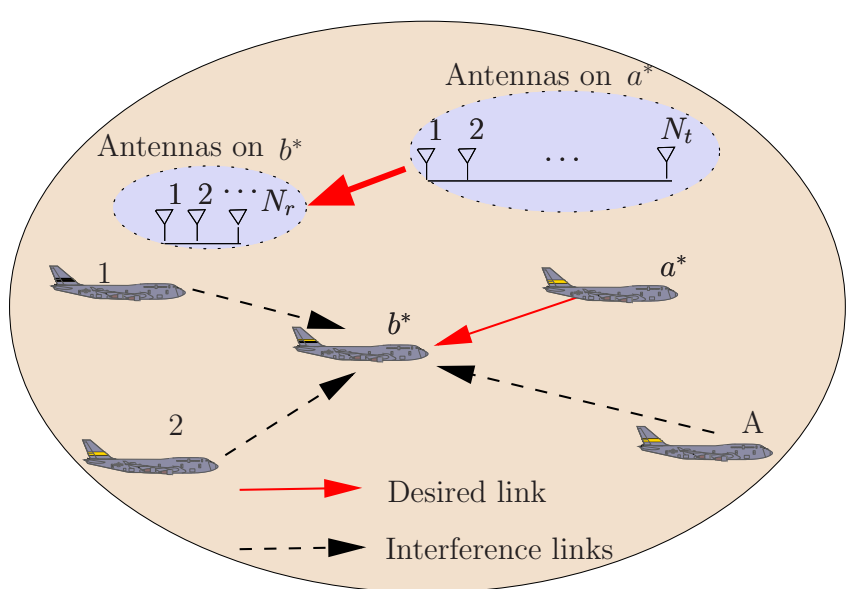

(b) Data transmission $\left(a^{*}\right.$ to $\left.b^{*}\right)$

Fig. 1. A TDD based aeronautical communication model with co-channel interference, where aircraft $a^{*}$ is transmitting data to aircraft $b^{*}$. 
asymptotic SINR in the presence of imperfect CSI and cochannel interference that leads to our detailed design of the achievable data rates and the associated switching distance thresholds. Section IV presents our simulation results for characterizing the impact of the relevant parameters in aeronautical communication. Our conclusions are given in Section V.

\section{SYSTEM MODEL}

Fig. 1 depicts an aeronautical communication system consisting of $(A+2)$ aircraft at their cruising altitudes within a given communication zone ${ }^{1}$. Aircraft $a^{*}$ and $b^{*}$ are the desired pair of communicating aircraft, with $a^{*}$ transmitting data to $b^{*}$, while aircraft $a=1,2, \cdots, A$ are interfering aircraft. As the system operates in SHF band, it is feasible to design compact high-gain antennas [25] and hence to deploy a largescale antenna array on an aircraft. This also enables every pair of communicating aircraft to use the same frequency and time slot. Specifically, the system is based on the TDD protocol, and each aircraft has $N_{\text {total }}$ antennas, which are capable of transmitting and receiving on the same frequency. Furthermore, from these $N_{\text {total }}$ antennas, $N_{t}$ antennas are utilized for transmitting data, while $N_{r}$ antennas are utilized for receiving data. We assume that the number of datareceiving antennas (DRAs) is no higher than that of the datatransmitting antennas (DTAs), i.e., $N_{r} \leq N_{t}<N_{\text {total }}$. The reasons are: 1) The spatial degrees of freedom $\min \left\{N_{r}, N_{t}\right\}$ determine the supportable data streams, and thus the number of DRAs should be no higher than the number of DTAs in order to make sure that the number of data streams after transmit precoding is no higher than $\min \left\{N_{r}, N_{t}\right\}$; and 2) The remaining $\left(N_{\text {total }}-N_{r}\right)$ or $\left(N_{\text {total }}-N_{t}\right)$ antennas are capable of transmitting/receiving other information, such as air traffic control or emergency information, at a frequency different from that of the data transmission. The system adopts orthogonal frequency-division multiplexing (OFDM) for mitigating the multipath effects and for achieving a high SE. We further assume that all the aircraft are jumbo jets, and they are all equipped with a same large-scale antenna array. We also assume that each aircraft has an airborne radar capable of measuring the distance to nearby aircraft. Alternatively, the distance information may be acquired with the aid of GPS.

As illustrated in Fig. 1 (b), aircraft $a^{*}$ employs $N_{t}$ antennas to transmit data and aircraft $b^{*}$ employs $N_{r}$ antennas to receive data. Therefore, $a^{*}$ has to know the multi-input multi-output (MIMO) channel matrix linking its $N_{t}$ DTAs to the $N_{r}$ DRAs of $b^{*}$ in order to carry out transmit precoding. The channel estimation is performed by pilot based training before data transmission, as shown in Fig. 1 (a). More specifically, aircraft $b^{*}$ transmits pilot symbols from its $N_{r}$ DRAs to aircraft $a^{*}$ 's $N_{t}$ DTAs to enable $a^{*}$ to perform the training based channel estimation. The required MIMO channel matrix can then be obtained by exploiting the TDD channel's reciprocity.

\section{A. Pilot based training}

During the pilot based training stage shown in Fig. 1(a), aircraft $b^{*}$ transmits its pilot symbols via its $N_{r}$ DRAs to

\footnotetext{
${ }^{1}$ Since Internet access is forbidden at takeoff and landing, it is reasonable to consider only aircraft en-route at cruising altitude.
}

the $N_{t}$ DTAs of aircraft $a^{*}$, and this pilot based training is interfered by all adjacent aircraft within the communication range. The most serious interference is imposed when the interfering aircraft $a=1,2, \cdots, A$ also transmit the same pilot symbols as aircraft $b^{*}$. The frequency domain (FD) MIMO channel between the $N_{r}$ DRAs of $b^{*}$ and the $N_{t}$ DTAs of $a^{*}$, on the $n$th OFDM subcarrier of the $s$ th symbol, is defined by the matrix $\boldsymbol{H}_{a^{*}}^{b^{*}}[s, n] \in \mathbb{C}^{N_{t} \times N_{r}}$, where $0 \leq n \leq N-1$ with $N$ denoting the length of the OFDM block. Let us assume that the length of the cyclic prefix (CP) $N_{\mathrm{cp}}$ is longer than the channel impulse response (CIR) $P$. The FD discrete signal model at the $n$th subcarrier and $s$ th time interval during the pilot training period can be written as

$$
\begin{aligned}
\widetilde{\boldsymbol{Y}}_{a^{*}} & {[s, n]=\sqrt{P_{r, a^{*}}^{b^{*}}} \boldsymbol{H}_{a^{*}}^{b^{*}}[s, n] \widetilde{\boldsymbol{X}}^{b^{*}}[s, n] } \\
& +\sum_{a=1}^{A} \sqrt{P_{r, a^{*}}^{a}} \boldsymbol{H}_{a^{*}}^{a}[s, n] \widetilde{\boldsymbol{X}}^{a}[s, n]+\widetilde{\boldsymbol{W}}_{a^{*}}[s, n],
\end{aligned}
$$

where $\tilde{\boldsymbol{Y}}_{a^{*}}[s, n]=\left[\widetilde{Y}_{1}^{a^{*}}[s, n] \widetilde{Y}_{2}^{a^{*}}[s, n] \cdots \widetilde{Y}_{N_{t}}^{a^{*}}[s, n]\right]^{\mathrm{T}} \in$ $\mathbb{C}^{N_{t} \times 1}$ represents the signal vector received by the $N_{t}$ DTAs of $a^{*}, \widetilde{\boldsymbol{X}}^{b^{*}}[s, n]=\left[\widetilde{X}_{1}^{b^{*}}[s, n] \widetilde{X}_{2}^{b^{*}}[s, n] \cdots \widetilde{X}_{N_{r}}^{b^{*}}[s, n]\right]^{\mathrm{T}} \in$ $\mathbb{C}^{N_{r} \times 1}$ is the transmitted pilot symbol vector, which obeys the complex white Gaussian distribution with a zero mean vector and the covariance matrix of $\boldsymbol{I}_{N_{r}}$, namely, $\widetilde{\boldsymbol{X}}^{b^{*}}[s, n] \sim$ $\mathcal{C N}\left(\mathbf{0}, \boldsymbol{I}_{N_{r}}\right)$, and $\widetilde{\boldsymbol{W}}_{a^{*}}[s, n] \sim \mathcal{C N}\left(\mathbf{0}, \sigma_{w}^{2} \boldsymbol{I}_{N_{t}}\right)$ is the associated FD additive white Gaussian noise (AWGN) vector, while the worst-case co-channel interference is considered with $\widetilde{\boldsymbol{X}}^{a}[s, n]=\widetilde{\boldsymbol{X}}^{b^{*}}[s, n]$ for $1 \leq a \leq A$. Here we have used $\mathbf{0}$ to represent the zero vector of an appropriate dimension and $\boldsymbol{I}_{N_{r}}$ to represent the $\left(N_{r} \times N_{r}\right)$-element identity matrix. Furthermore, in (1), $P_{r, a^{*}}^{b^{*}}$ and $P_{r, a^{*}}^{a}$ denote the received powers of the desired signal and the interference signal transmitted from $b^{*}$ and $a$ at a single receive antenna, respectively.

The FD channel transfer function coefficient (FD-CHTFC) matrix $\boldsymbol{H}_{a^{*}}^{b^{*}}[s, n]$ is explicitly given by

$$
\boldsymbol{H}_{a^{*}}^{b^{*}}[s, n]=\nu \boldsymbol{H}_{\mathrm{d}, a^{*}}^{b^{*}}[s, n]+\varsigma \boldsymbol{H}_{\mathrm{r}, a^{*}}^{b^{*}}[s, n],
$$

where $\boldsymbol{H}_{\mathrm{d}, a^{*}}^{b^{*}}[s, n] \in \mathbb{C}^{N_{t} \times N_{r}}$ and $\boldsymbol{H}_{\mathrm{r}, a^{*}}^{b^{*}}[s, n] \in \mathbb{C}^{N_{t} \times N_{r}}$ denote the deterministic and scattered channel components, respectively, while $\nu=\sqrt{\frac{K_{\text {Rice }}}{K_{\text {Rice }}+1}}$ and $\varsigma=\sqrt{\frac{1}{K_{\text {Rice }}+1}}$, with $K_{\text {Rice }}$ being the $K$-factor of the Rician channel. Furthermore, the scattered channel component is given by [26]

$$
\boldsymbol{H}_{\mathrm{r}, a^{*}}^{b^{*}}[s, n]=\boldsymbol{R}_{a^{*}}^{\frac{1}{2}} \boldsymbol{G}_{a^{*}}^{b^{*}}[s, n] \boldsymbol{R}^{b^{*} \frac{1}{2}},
$$

where $\boldsymbol{R}_{a^{*}} \in \mathbb{C}^{N_{t} \times N_{t}}$ and $\boldsymbol{R}^{b^{*}} \in \mathbb{C}^{N_{r} \times N_{r}}$ are the spatial correlation matrices for the $N_{t}$ antennas of $a^{*}$ and the $N_{r}$ antennas of $b^{*}$, respectively, while $G_{a^{*}}^{b^{*}}[s, n] \in \mathbb{C}^{N_{t} \times N_{r}}$ has the independently identically distributed complex-valued entries, each obeying the distribution $\mathcal{C N}(0,1)$. As a benefit of the CP, the OFDM symbols do not overlap in time and the processing can be carried out on a per-carrier basis [27]. Hence, to simplify our notations, we will omit the OFDM symbol index $s$ and the subcarrier index $n$ in the sequel.

Let $\boldsymbol{v e c}(\boldsymbol{A})$ denote the column stacking operation applied to matrix $\boldsymbol{A}$. Clearly, $\mathcal{E}\left\{\boldsymbol{v} \boldsymbol{e c}\left(\boldsymbol{H}_{\mathrm{r}, a^{*}}^{b^{*}}\right)\right\}=0$, where $\mathcal{E}\{\}$ 


$$
\begin{aligned}
& \boldsymbol{v e c}\left(\widehat{\boldsymbol{H}}_{a^{*}}^{b^{*}}\right)=\boldsymbol{v} \boldsymbol{e c}\left(\nu \boldsymbol{H}_{\mathrm{d}, a^{*}}^{b^{*}}\right)+\varsigma^{2} \overline{\boldsymbol{R}}_{\mathrm{r}, t}^{r}\left(\frac{\sigma_{w}^{2}}{P_{r, a^{*}}^{b^{*}}} \boldsymbol{I}_{N_{r} N_{t}}+\varsigma^{2} \overline{\boldsymbol{R}}_{\mathrm{r}, t}^{r}+\sum_{a=1}^{A} \frac{P_{r, a^{*}}^{a}}{P_{r, a^{*}}^{b^{*}}} \varsigma^{2} \overline{\boldsymbol{R}}_{\mathrm{r}, t}^{r}\right)^{-1} \\
& \times\left(\boldsymbol{v e c}\left(\varsigma \boldsymbol{H}_{\mathrm{r}, a^{*}}^{b^{*}}\right)+\sum_{a=1}^{A} \sqrt{\frac{P_{r, a^{*}}^{a}}{P_{r, a^{*}}^{b^{*}}}} \boldsymbol{v} \operatorname{ec}\left(\varsigma \boldsymbol{H}_{\mathrm{r}, a^{*}}^{a}\right)+\frac{1}{\sqrt{P_{r, a^{*}}^{b^{*}}}} \boldsymbol{v e c}\left(\widetilde{\overline{\boldsymbol{W}}}_{a^{*}}\left(\widetilde{\overline{\boldsymbol{X}}}^{b^{*}}\right)^{\mathrm{H}}\right)\right) .
\end{aligned}
$$

denotes the expectation operator, and the covariance matrix $\boldsymbol{R}_{\mathrm{r}, a^{*}}^{b^{*}} \in \mathbb{C}^{N_{t} N_{r} \times N_{t} N_{r}}$ of $\boldsymbol{v} \boldsymbol{e c}\left(\boldsymbol{H}_{\mathrm{r}, a^{*}}^{b^{*}}\right)$ is given by

$$
\boldsymbol{R}_{\mathrm{r}, a^{*}}^{b^{*}}=\mathcal{E}\left\{\boldsymbol{v e c}\left(\boldsymbol{H}_{\mathrm{r}, a^{*}}^{b^{*}}\right)\left(\boldsymbol{v e c}\left(\boldsymbol{H}_{\mathrm{r}, a^{*}}^{b^{*}}\right)\right)^{\mathrm{H}}\right\}=\boldsymbol{R}^{b^{*}} \otimes \boldsymbol{R}_{a^{*}}
$$

where $\otimes$ denotes the matrix Kronecker product. Therefore, $\operatorname{vec}\left(\boldsymbol{H}_{a^{*}}^{b^{*}}\right) \sim \mathcal{C N}\left(\boldsymbol{v e c}\left(\nu \boldsymbol{H}_{\mathrm{d}, a^{*}}^{b^{*}}\right), \boldsymbol{R}_{\mathrm{r}, a^{*}}^{b^{*}}\right)$. Since all the aircraft have the same antenna array, we make the assumption that all the spatial correlation matrices $\boldsymbol{R}_{a_{t}}$ for $a_{t} \in \mathcal{A}=$ $\left\{1,2, \cdots, A, a^{*}, b^{*}\right\}$ are approximately equal, i.e., we assume that $\boldsymbol{R}_{a_{t}}=\overline{\boldsymbol{R}}_{t} \forall a_{t} \in \mathcal{A}$ hold $^{2}$. By the same argument, we assume that $\boldsymbol{R}^{a_{r}}=\overline{\boldsymbol{R}}^{r} \forall a_{r} \in \mathcal{A}$ hold $^{3}$. Thus, all the channels' covariance matrices are assumed to be equal, i.e., we have

$$
\boldsymbol{R}_{\mathrm{r}, a_{t}}^{a_{r}}=\overline{\boldsymbol{R}}_{\mathrm{r}, t}^{r}=\overline{\boldsymbol{R}}^{r} \otimes \overline{\boldsymbol{R}}_{t}, \forall a_{t}, a_{r} \in \mathcal{A} \text { and } a_{t} \neq a_{r} .
$$

This implies that every aircraft has the knowledge of its channel covariance matrix. For example, aircraft $a^{*}$ knows its antenna array's spatial correlation matrices $\boldsymbol{R}_{a^{*}}=\overline{\boldsymbol{R}}_{t}$ and $\boldsymbol{R}^{a^{*}}=\overline{\boldsymbol{R}}^{r}$, and since $\boldsymbol{R}^{b^{*}}=\boldsymbol{R}^{a^{*}}$, it knows its channel covariance matrix $\boldsymbol{R}_{\mathrm{r}, a^{*}}^{b^{*}}=\boldsymbol{R}^{b^{*}} \otimes \boldsymbol{R}_{a^{*}}=\boldsymbol{R}^{a^{*}} \otimes \boldsymbol{R}_{a^{*}}=\overline{\boldsymbol{R}}^{r} \otimes \overline{\boldsymbol{R}}_{t}$. It can be seen that $\boldsymbol{R}^{b^{*}}=\boldsymbol{R}^{a^{*}}$ is the real assumption required ${ }^{4}$.

The received power $P_{r, a^{*}}^{b^{*}}$ at aircraft $a^{*}$ is linked to the transmitted signal power $P_{t}^{b^{*}}$ of aircraft $b^{*}$ by the following path loss model [25]

$$
P_{r, a^{*}}^{b^{*}}=P_{t}^{b^{*}} 10^{-0.1 L_{\text {path loss }, a^{*}}^{b^{*}},}
$$

where $L_{\text {path loss, } a^{*}}^{b^{*}}$ represents the path loss in $\mathrm{dB}$, which can be modeled as [25]

$$
L_{\text {path loss }, a^{*}}^{b^{*}}[\mathrm{~dB}]=-154.06+20 \log _{10}(f)+20 \log _{10}(d),
$$

where $f[\mathrm{~Hz}]$ is the carrier frequency and $d[\mathrm{~m}]$ is the distance between the transmit antenna and the receive antenna. For the received interference signal power $P_{r, a^{*}}^{a}$, we have a similar path loss model. Each entry of the FD AWGN vector $\boldsymbol{W}_{a^{*}}$ obeys the distribution $\mathcal{C N}\left(0, \sigma_{w}^{2}\right)$ with $\sigma_{w}^{2}=\frac{P_{N}}{N}$, in which $P_{N}$ is the receiver noise power given by [28]

$$
P_{N}=F k T_{0} B
$$

where $F[\mathrm{~dB}]$ is the receiver's noise figure, $T_{0}$ is the reference temperature in Kelvin at the receiver, $k=1.3 \times 10^{-23}$ is Boltzmann's constant and $B[\mathrm{~Hz}]$ is the bandwidth.

\footnotetext{
${ }^{2}$ It is reasonable to assume that all jumbo jets are equipped with identical antenna array. However, because the geometric shapes of different types of jumbo jets are slightly different, $\boldsymbol{R}_{a_{t}}=\overline{\boldsymbol{R}}_{t} \forall a_{t} \in \mathcal{A}$ only hold approximately.

${ }^{3}$ Similarly, $\boldsymbol{R}^{a_{r}}=\overline{\boldsymbol{R}}^{r} \forall a_{r} \in \mathcal{A}$ only hold approximately.

${ }^{4}$ Alternatively, we can also avoid imposing this assumption. Then, $a^{*}$ can ask $b^{*}$ to send its antenna correlation matrix $\boldsymbol{R}^{b^{*}}$. For example, during the initial handshake of establishing the link, $b^{*}$ can sends $\boldsymbol{R}^{b^{*}}$ to $a^{*}$ through the signaling at the expense of increasing the signaling overhead.
}

Since every aircraft knows its channel covariance matrix, we can apply the optimal minimum mean square error (MMSE) estimator [29] to estimate the channel matrix $\boldsymbol{H}_{a^{*}}^{b^{*}}$. The MMSE estimate $\widehat{\boldsymbol{H}}_{b^{*}}^{b^{*}}{ }^{*}$ of $\boldsymbol{H}_{a^{*}}^{b^{*}}$ is given in (9) at the top of this page, where $\widetilde{\overline{\boldsymbol{X}}} b^{*} \in \mathbb{C}^{N_{r} \times N_{r}}$ consists of the $N_{r}$ pilot symbols with $\widetilde{\overline{\boldsymbol{X}}}^{b^{*}}\left(\widetilde{\overline{\boldsymbol{X}}}^{b^{*}}\right)^{\mathrm{H}}=\boldsymbol{I}_{N_{r}}$, and $\widetilde{\overline{\boldsymbol{W}}}_{a^{*}} \in \mathbb{C}^{N_{r} \times N_{r}}$ is the corresponding AWGN vector over the $N_{r}$ consecutive OFDM pilot symbols. It is well-known that the distribution of the MMSE estimator (9) is $\operatorname{vec}\left(\widehat{\boldsymbol{H}}_{a^{*}}^{b^{*}}\right) \sim \mathcal{C N}\left(\boldsymbol{v e c}\left(\nu \boldsymbol{H}_{\mathrm{d}, a^{*}}^{b^{*}}\right), \boldsymbol{\Phi}_{a^{*}}^{b^{*}}\right)$ [29], that is, $\boldsymbol{v e c}\left(\widehat{\boldsymbol{H}}_{a^{*}}^{b^{*}}\right)$ is an unbiased estimate of $\boldsymbol{v e c}\left(\boldsymbol{H}_{a^{*}}^{b^{*}}\right)$ with the estimation accuracy specified by the covariance matrix $\boldsymbol{\Phi}_{a^{*}}^{b^{*}} \in$ $\mathbb{C}^{N_{t} N_{r} \times N_{t} N_{r}}$, which is given by

$$
\begin{aligned}
\boldsymbol{\Phi}_{a^{*}}^{b^{*}}= & \varsigma^{2} \overline{\boldsymbol{R}}_{\mathrm{r}, t}^{r}\left(\frac{\sigma_{w}^{2}}{P_{r, a^{*}}^{b^{*}}} \boldsymbol{I}_{N_{r} N_{t}}+\varsigma^{2} \overline{\boldsymbol{R}}_{\mathrm{r}, t}^{r}+\sum_{a=1}^{A} \frac{P_{r, a^{*}}^{a}}{P_{r, a^{*}}^{b^{*}}} \varsigma^{2} \overline{\boldsymbol{R}}_{\mathrm{r}, t}^{r}\right)^{-1} \\
& \times \varsigma^{2} \overline{\boldsymbol{R}}_{\mathrm{r}, t}^{r} .
\end{aligned}
$$

\section{B. Data transmission}

During the data transmission, aircraft $a^{*}$ transmits the symbols $\boldsymbol{X}^{a^{*}}=\left[\begin{array}{ll}X_{1}^{a *} & X_{2}^{a *} \cdots X_{N_{r}}^{a *}\end{array}\right]^{\mathrm{T}} \in \mathbb{C}^{N_{r} \times 1}$ from its $N_{t}$ DTAs to the $N_{r}$ DRAs of aircraft $b^{*}$. Let us denote the MIMO channel matrix during this data transmission as $\boldsymbol{H}_{b^{*}}^{a^{*}} \in$ $\mathbb{C}^{N_{r} \times N_{t}}$. Then, upon exploiting the channel's reciprocity in TDD systems, we have $\boldsymbol{H}_{b^{*}}^{a^{*}}=\left(\boldsymbol{H}_{a^{*}}^{b^{*}}\right)^{\mathrm{H}}$.

To mitigate the interference between multiple antennas, transmit precoding (TPC) is adopted for data transmission. There are various methods of designing the TPC matrix $\boldsymbol{V}_{b^{*}}^{a^{*}} \in \mathbb{C}^{N_{t} \times N_{r}}$, including the convex optimization based method of [30], the minimum variance method of [31], the minimum bit-error rate (MBER) design of [32], the MMSE design of [33] and the zero-forcing (ZF) design as well as the eigen-beamforming or matched filter (MF) design of [34]. For a large-scale MIMO system, the complexity of the optimization based TPC designs of [30]-[32] may become excessive. Additionally, in this case, the performance of the MBER design [32] is indistinguishable from the MMSE one. Basically, for large-scale antenna array based MIMO, the performance of the MMSE, ZF and MF based TPC solutions are sufficiently good. The MF TPC design offers the additional advantage of the lowest complexity and, therefore, it is chosen in this work. Specifically, the MF TPC matrix is given by

$$
\boldsymbol{V}_{b^{*}}^{a^{*}}=\left(\widehat{\boldsymbol{H}}_{b^{*}}^{a^{*}}\right)^{\mathrm{H}}=\widehat{\boldsymbol{H}}_{a^{*}}^{b^{*}},
$$

where $\widehat{\boldsymbol{H}}_{b^{*}}^{a^{*}}$ denotes the estimate of $\boldsymbol{H}_{b^{*}}^{a^{*}}$, and $\widehat{\boldsymbol{H}}_{a^{*}}^{b^{*}}$ is the channel estimate obtained during pilot training. 


$$
\begin{aligned}
& Y_{n_{r}^{*}}^{b^{*}}=\sqrt{P_{r, b^{*}}^{a^{*}}}\left[\boldsymbol{H}_{b^{*}}^{a^{*}}\right]_{\left[n_{r}^{*}:\right]} \boldsymbol{V}_{b^{*}}^{a^{*}} \boldsymbol{X}^{a^{*}}+\sum_{a=1}^{A} \sqrt{P_{r, b^{*}}^{a}}\left[\boldsymbol{H}_{b^{*}}^{a}\right]_{\left[n_{r}^{*}:\right]} \boldsymbol{V}_{b^{a}}^{a} \boldsymbol{X}^{a}+W_{n_{r}^{*}}^{b^{*}}=\sqrt{P_{r, b^{*}}^{a^{*}}}\left[\boldsymbol{H}_{b^{*}}^{a^{*}}\right]_{\left[n_{r}^{*}:\right]}\left[\boldsymbol{V}_{b^{*}}^{a^{*}}\right]_{\left[: n_{r}^{*}\right]} X_{n_{r}^{*}}^{a^{*}} \\
& +\sum_{n_{r} \neq n_{r}^{*}} \sqrt{P_{r, b^{*}}^{a^{*}}}\left[\boldsymbol{H}_{b^{*}}^{a^{*}}\right]_{\left[n_{r}^{*}:\right]}\left[\boldsymbol{V}_{b^{*}}^{a^{*}}\right]_{\left[: n_{r}\right]} X_{n_{r}}^{a^{*}}+\sum_{a=1}^{A} \sum_{n_{r}=1}^{N_{r}} \sqrt{P_{r, b^{*}}^{a}}\left[\boldsymbol{H}_{b^{*}}^{a}\right]_{\left[n_{r}^{*}:\right]}\left[\boldsymbol{V}_{b^{a}}^{a}\right]_{\left[: n_{r}\right]} X_{n_{r}}^{a}+W_{n_{r}^{*}}^{b^{*}}, \\
& Y_{n_{r}^{*}}^{b^{*}}=\mathcal{E}\left\{\sqrt{P_{r, b^{*}}^{a^{*}}}\left[\boldsymbol{H}_{b^{*}}^{a^{*}}\right]_{\left[n_{r}^{*}:\right]}\left[\boldsymbol{V}_{b^{*}}^{a^{*}}\right]_{\left[: n_{r}^{*}\right]}\right\} X_{n_{r}^{*}}^{a^{*}}+\left(\sqrt{P_{r, b^{*}}^{a^{*}}}\left[\boldsymbol{H}_{b^{*}}^{a^{*}}\right]_{\left[n_{r}^{*}:\right]}\left[\boldsymbol{V}_{b^{*}}^{a^{*}}\right]_{\left[: n_{r}^{*}\right]}-\mathcal{E}\left\{\sqrt{P_{r, b^{*}}^{a^{*}}}\left[\boldsymbol{H}_{b^{*}}^{a^{*}}\right]_{\left[n_{r}^{*}:\right]}\left[\boldsymbol{V}_{b^{*}}^{a^{*}}\right]_{\left[: n_{r}^{*}\right]}\right\}\right) X_{n_{r}^{*}}^{a^{*}} \\
& +\sum_{n_{r} \neq n_{r}^{*}} \sqrt{P_{r, b^{*}}^{a^{*}}}\left[\boldsymbol{H}_{b^{*}}^{a^{*}}\right]_{\left[n_{r}^{*}:\right]}\left[\boldsymbol{V}_{b^{*}}^{a^{*}}\right]_{\left[: n_{r}\right]} X_{n_{r}}^{a^{*}}+\sum_{a=1}^{A} \sum_{n_{r}=1}^{N_{r}} \sqrt{P_{r, b^{*}}^{a}}\left[\boldsymbol{H}_{b^{*}}^{a}\right]_{\left[n_{r}^{*}:\right]}\left[\boldsymbol{V}_{b^{a}}^{a}\right]_{\left[: n_{r}\right]} X_{n_{r}}^{a}+W_{n_{r}^{*}}^{b^{*}} \text {. }
\end{aligned}
$$

In the presence of the interference imposed by aircraft $a$ for $1 \leq a \leq A$, the received signal vector at aircraft $b^{*}, \boldsymbol{Y}_{b^{*}}=$ $\left[Y_{1}^{b^{*}} Y_{2}^{b^{*}} \cdots Y_{N_{r}}^{b^{*}}\right]^{\mathrm{T}} \in \mathbb{C}^{N_{r} \times 1}$, can be written as

$$
\boldsymbol{Y}_{b^{*}}=\sqrt{P_{r, b^{*}}^{a^{*}}} \boldsymbol{H}_{b^{*}}^{a^{*}} \boldsymbol{V}_{b^{*}}^{a^{*}} \boldsymbol{X}^{a^{*}}+\sum_{a=1}^{A} \sqrt{P_{r, b^{*}}^{a}} \boldsymbol{H}_{b^{*}}^{a} \boldsymbol{V}_{b^{a}}^{a} \boldsymbol{X}^{a}+\boldsymbol{W}_{b^{*}},
$$

where $\boldsymbol{V}_{b^{a}}^{a} \in \mathbb{C}^{N_{t} \times N_{r}}$ denotes the TPC matrix at aircraft $a$ transmitting the signal $\boldsymbol{X}^{a}=\left[X_{1}^{a} X_{2}^{a} \cdots X_{N_{r}}^{a}\right]^{\mathrm{T}}$ to its desired receiving aircraft $b^{a}$ for $b^{a} \neq b^{*}$, and the channel's AWGN vector is $\boldsymbol{W}_{b^{*}}=\left[W_{1}^{b^{*}} W_{2}^{b^{*}} \cdots W_{N_{r}}^{b^{*}}\right]^{\mathrm{T}} \sim \mathcal{C N}\left(\mathbf{0}, \sigma_{w}^{2} \boldsymbol{I}_{N_{r}}\right)$. In particular, the signal received at the antenna $n_{r}^{*}$ of aircraft $b^{*}$, for $1 \leq n_{r}^{*} \leq N_{r}$, is given by (13) at the top of this page, where $[\boldsymbol{A}]_{\left[n_{r}:\right]} \in \mathbb{C}^{1 \times M}$ denotes the $n_{r}$ th row of $\boldsymbol{A} \in \mathbb{C}^{N \times M}$ and $[\boldsymbol{A}]_{\left[: n_{r}\right]} \in \mathbb{C}^{N \times 1}$ denotes the $n_{r}$ th column of $\boldsymbol{A}$.

\section{Proposed ADAPTIVE Coding AND MOdulation}

In order to attain the required high-throughput transmission for our large-scale antenna assisted aeronautical communication system, we propose a distance-based ACM scheme. We begin by analyzing the system's achievable throughput, followed by the detailed design of this distance-based ACM.

\section{A. Achievable throughput analysis}

The transmitting aircraft $a^{*}$ pre-codes its signals based on the channel estimate obtained during the pilot training by exploiting the TDD channel's reciprocity. However, the receiving aircraft $b^{*}$ does not have this CSI. Thus, the ergodic achievable rate is adopted for analyzing the achievable throughput. In order to explicitly derive this capacity, we rewrite the received signal at the antenna $n_{r}^{*}$ of aircraft $b^{*}$, namely, $Y_{n_{r}^{*}}^{b^{*}}$ of (13), in the form given in (14). Observe that the first term of (14) is the desired signal, the second term is the interference caused by the channel estimation error, the third term is the interference arriving from the other antennas of $b^{*}$, and the fourth term is the interference impinging from the interfering aircraft, while the last term is of course the noise. Thus, the SINR at the $n_{r}$-th antenna of $b^{*}$, denoted by $\gamma_{b^{*}, n_{r}}^{a^{*}}$, is given by

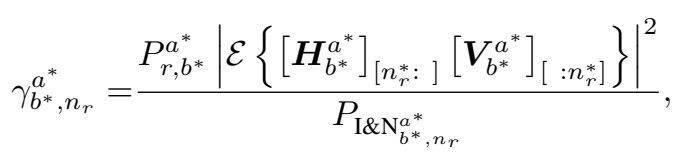

in which the power of the interference plus noise is

$$
\begin{array}{r}
P_{\mathrm{I \& N} \mathrm{N}_{b^{*}, n_{r}}^{a^{*}}}=\sigma_{w}^{2}+P_{r, b^{*}}^{a^{*}} \operatorname{Var}\left\{\left[\boldsymbol{H}_{b^{*}}^{\left.a^{*}\right]_{\left[n_{r}^{*}:\right]}}\left[\boldsymbol{V}_{b^{*}}^{\left.a^{*}\right]}\right]_{\left[: n_{r}^{*}\right]}\right\}\right. \\
+P_{r, b^{*}}^{a^{*}} \sum_{n_{r} \neq n_{r}^{*}} \mathcal{E}\left\{\left|\left[\boldsymbol{H}_{b^{*}}^{a^{*}}\right]_{\left[n_{r}^{*}:\right]}\left[\boldsymbol{V}_{b^{*}}^{a^{*}}\right]_{\left[: n_{r}\right]}\right|^{2}\right\} \\
+\sum_{a=1}^{A} P_{r, b^{*}}^{a} \sum_{n_{r}=1}^{N_{r}} \mathcal{E}\left\{\left|\left[\boldsymbol{H}_{b^{*}}^{a}\right]_{\left[n_{r}^{*}:\right]}\left[\boldsymbol{V}_{b^{a}}^{a}\right]_{\left[: n_{r}\right]}\right|^{2}\right\},
\end{array}
$$

where $\operatorname{Var}\{\}$ denoting the variance. The achievable transmission rate per antenna between the transmitting aircraft $a^{*}$ and destination aircraft $b^{*}$ can readily be obtained as

$$
C_{b^{*}}^{a^{*}}=\frac{1}{N_{r}} \sum_{n_{r}=1}^{N_{r}} \log _{2}\left(1+\gamma_{b^{*}, n_{r}}^{a^{*}}\right) .
$$

As mentioned previously, the distance between the transmitting aircraft and the receiving aircraft is available with the aid of airborne radar or GPS. But we do not require that the distances between the interfering aircraft and the desired destination aircraft are known to the transmitting aircraft. Realistically, the distance between two aircraft can be assumed to follow the uniform distribution within the range of $\left[D_{\min }, D_{\max }\right]$, where $D_{\min }$ is the minimum separation distance required by safety and $D_{\max }$ is the maximum communication range [35]. For example, we have $D_{\max }=$ 400 nautical miles, which is approximately $740.8 \mathrm{~km}$, for a typical cruising altitude of $10.68 \mathrm{~km}$. Normally, the International Civil Aviation Organization prescribes the minimum separation as 5 nautical miles (approximately $9.26 \mathrm{~km}$ ) when surveillance systems are in use. But the minimum separation distance could be reduced to 2.5 nautical miles (about 4.63 $\mathrm{km})$ when surveillance radars are intensively deployed, such as in an airport's airspace. Thus, the minimum distance is set to $D_{\min }=5 \mathrm{~km}$ in the envisaged AANET. Intuitively, an aircraft always transmits its signal to an aircraft having the best propagation link with it for relying its information in the AANET. Here we simply assume that a pair of aircraft having the shortest communication distance have the best propagation link, since large-scale fading dominates the quality of propagation in aeronautical communication. Being in mind (6) and (7) as well as the fact that the distance $d$ is uniformly distributed in $\left[D_{\min }, D_{\max }\right]$, we can express the average received signal 
power for the transmission from aircraft $a$ to aircraft $b^{*}$ as

$$
\bar{P}_{r}=\bar{P}_{r, b^{*}}^{a}=\mathcal{E}\left\{P_{r, b^{*}}^{a}\right\}=P_{t}^{a} \frac{10^{15.406}}{f^{2}} \frac{1}{D_{\max } D_{\min }} .
$$

The relationship between the MMSE estimate $\left[\widehat{\boldsymbol{H}}_{b^{*}}^{a^{*}}\right]_{\left[n_{r}:\right]}$ and the true MIMO channel $\left[\boldsymbol{H}_{b^{*}}^{a^{*}}\right]_{\left[n_{r}:\right]}$ can be expressed as

$$
\left[\boldsymbol{H}_{b^{*}}^{a^{*}}\right]_{\left[n_{r}:\right]}=\left[\widehat{\boldsymbol{H}}_{b^{*}}^{a^{*}}\right]_{\left[n_{r}:\right]}+\left[\widetilde{\boldsymbol{H}}_{b^{*}}^{a^{*}}\right]_{\left[n_{r}:\right]},
$$

where $\left[\widetilde{\boldsymbol{H}}_{b^{*}}^{a^{*}}\right]_{\left[n_{r}:\right]}$ denotes the estimation error, which is statistically independent of both $\left[\widehat{\boldsymbol{H}}_{b^{*}}^{a^{*}}\right]_{\left[n_{r}:\right]}$ and $\left[\boldsymbol{H}_{b^{*}}^{a^{*}}\right]_{\left[n_{r}:\right]}$ [29]. Similar to the distribution of $\boldsymbol{v e c}\left(\widehat{\boldsymbol{H}}_{a^{*}}^{b^{*}}\right)$, we have

$$
\boldsymbol{v e c}\left(\widehat{\boldsymbol{H}}_{b^{*}}^{a^{*}}\right) \sim \mathcal{C N}\left(\boldsymbol{v e c}\left(\nu \boldsymbol{H}_{\mathrm{d}, b^{*}}^{a^{*}}\right), \boldsymbol{\Phi}_{b^{*}}^{a^{*}}\right),
$$

where the covariance matrix $\boldsymbol{\Phi}_{b^{*}}^{a^{*}}$ of the MMSE estimate $\operatorname{vec}\left(\widehat{\boldsymbol{H}}_{b^{*}}^{a^{*}}\right)$ is given by.

$$
\begin{aligned}
\boldsymbol{\Phi}_{b^{*}}^{a^{*}}= & \varsigma^{2} \overline{\boldsymbol{R}}_{\mathrm{r}, r}^{t}\left(\frac{\sigma_{w}^{2}}{P_{r, b^{*}}^{a^{*}}} \boldsymbol{I}_{N_{r} N_{t}}+\varsigma^{2} \overline{\boldsymbol{R}}_{\mathrm{r}, r}^{t}+\sum_{a=1}^{A} \frac{P_{r, a^{*}}^{a}}{P_{r, b^{*}}^{a^{*}}} \varsigma^{2} \overline{\boldsymbol{R}}_{\mathrm{r}, r}^{t}\right)^{-1} \\
& \times \varsigma^{2} \overline{\boldsymbol{R}}_{\mathrm{r}, r}^{t},
\end{aligned}
$$

in which $\varsigma^{2} \overline{\boldsymbol{R}}_{\mathrm{r}, r}^{t}$ is the channel's covariance matrix and the channel's spatial correlation matrix $\overline{\boldsymbol{R}}_{\mathrm{r}, r}^{t}$ is given by

$$
\overline{\boldsymbol{R}}_{\mathrm{r}, r}^{t}=\overline{\boldsymbol{R}}_{t} \otimes \overline{\boldsymbol{R}}^{r}
$$

Denote the covariance matrix of the channel estimation error $\operatorname{vec}\left(\widetilde{\boldsymbol{H}}_{b^{*}}^{a^{*}}\right)=\operatorname{vec}\left(\boldsymbol{H}_{b^{*}}^{a^{*}}\right)-\boldsymbol{v e c}\left(\widehat{\boldsymbol{H}}_{b^{*}}^{a^{*}}\right)$ by $\boldsymbol{\Xi}_{b^{*}}^{a^{*}}$. Clearly, we have

$$
\boldsymbol{\Xi}_{b^{*}}^{a^{*}}=\varsigma^{2} \overline{\boldsymbol{R}}_{\mathrm{r}, r}^{t}-\boldsymbol{\Phi}_{b^{*}}^{a^{*}} \in \mathbb{C}^{N_{t} N_{r} \times N_{t} N_{r}},
$$

and $\boldsymbol{\Xi}_{b^{*}}^{a^{*}}$ can be explicitly expressed in the following form

$$
\boldsymbol{\Xi}_{b^{*}}^{a^{*}}=\left[\begin{array}{cccc}
{\left[\boldsymbol{\Xi}_{b^{*}}^{a^{*}}\right]_{(1,1)}} & {\left[\boldsymbol{\Xi}_{b^{*}}^{a^{*}}\right]_{(1,2)}} & \cdots & {\left[\boldsymbol{\Xi}_{b^{*}}^{a^{*}}\right]_{\left(1, N_{r}\right)}} \\
\vdots & \vdots & \cdots & \vdots \\
{\left[\boldsymbol{\Xi}_{b^{*}}^{a^{*}}\right]_{\left(N_{r}, 1\right)}} & {\left[\boldsymbol{\Xi}_{b^{*}}^{a^{*}}\right]_{\left(N_{r}, 2\right)}} & \cdots & {\left[\boldsymbol{\Xi}_{b^{*}}^{a^{*}}\right]_{\left(N_{r}, N_{r}\right)}}
\end{array}\right],
$$

where $\left[\boldsymbol{\Xi}_{b^{*}}^{a^{*}}\right]_{(i, j)}=\mathcal{E}\left\{\left[\widetilde{\boldsymbol{H}}_{b^{*}}^{a^{*}}\right]_{[i:]}^{\mathrm{H}}\left[\widetilde{\boldsymbol{H}}_{b^{*}}^{a^{*}}\right]_{[j:]}\right\} \in \mathbb{C}^{N_{t} \times N_{t}}$, $\forall i, j \in\left\{1,2, \cdots, N_{r}\right\}$. This indicates that the distribution of $\left[\widetilde{\boldsymbol{H}}_{b^{*}}^{a^{*}}\right]_{\left[n_{r}:\right]}$ is given by

$$
\left[\widetilde{\boldsymbol{H}}_{b^{*}}^{a^{*}}\right]_{\left[n_{r}:\right]}^{\mathrm{T}} \sim \mathcal{C N}\left(\mathbf{0}_{N_{t} \times 1},\left[\boldsymbol{\Xi}_{b^{*}}^{a^{*}}\right]_{\left(n_{r}, n_{r}\right)}\right),
$$

where $\mathbf{0}_{N_{t} \times 1}$ is the $N_{t}$-dimensional zero vector.

Noting the distribution (20), the correlation matrix obeys $\mathcal{E}\left\{\boldsymbol{v e c}\left(\widehat{\boldsymbol{H}}_{a^{*}}^{b^{*}}\right) \boldsymbol{v e c}\left(\widehat{\boldsymbol{H}}_{a^{*}}^{b^{*}}\right)^{\mathrm{H}}\right\}=\nu^{2} \boldsymbol{M}_{b^{*}}^{a^{*}}+\boldsymbol{\Phi}_{b^{*}}^{a^{*}}$, where

$$
\boldsymbol{M}_{b^{*}}^{a^{*}}=\boldsymbol{v e c}\left(\boldsymbol{H}_{\mathrm{d}, a^{*}}^{b^{*}}\right) \boldsymbol{v e c}\left(\boldsymbol{H}_{\mathrm{d}, a^{*}}^{b^{*}}\right)^{\mathrm{H}} \in \mathbb{C}^{N_{t} N_{r} \times N_{t} N_{r}} .
$$

Furthermore, $\boldsymbol{M}_{b^{*}}^{a^{*}}$ can be expressed in a form similar to (24) having the $(i, j)$-th sub-matrix $\left[\boldsymbol{M}_{b^{*}}^{a^{*}}\right]_{(i, j)}=$ $\left[\boldsymbol{H}_{\mathrm{d}, a^{*}}^{b^{*}}\right]_{[i:]}^{\mathrm{H}}\left[\boldsymbol{H}_{\mathrm{d}, a^{*}}^{b^{*}}\right]_{[j:]} \in \mathbb{C}^{N_{t} \times N_{t}}, \forall i, j \in\left\{1,2, \cdots, N_{r}\right\}$.
Hence, upon recalling (11) and (19), we have

$$
\begin{aligned}
\mathcal{E}\left\{\left[\boldsymbol{H}_{b^{*}}^{a^{*}}\right]_{\left[n_{r}^{*}:\right]}\left[\boldsymbol{V}_{b^{*}}^{a^{*}}\right]_{\left[: n_{r}^{*}\right]}\right\}=\mathcal{E}\left\{\left[\boldsymbol{H}_{b^{*}}^{a^{*}}\right]_{\left[n_{r}^{*}:\right]}\left[\widehat{\boldsymbol{H}}_{b^{*}}^{a^{*}}\right]_{\left[n_{r}^{*}:\right]}^{\mathrm{H}}\right\} \\
=\mathcal{E}\left\{\left(\left[\widehat{\boldsymbol{H}}_{b^{*}}^{a^{*}}\right]_{\left[n_{r}^{*}:\right]}+\left[\widetilde{\boldsymbol{H}}_{b^{*}}^{a^{*}}\right]_{\left[n_{r}^{*}:\right]}\right)\left[\widehat{\boldsymbol{H}}_{b^{*}}^{a^{*}}\right]_{\left[n_{r}^{*}:\right]}^{\mathrm{H}}\right\} \\
=\mathcal{E}\left\{\operatorname{Tr}\left\{\left[\widehat{\boldsymbol{H}}_{b^{*}}^{a^{*}}\right]_{\left[n_{r}^{*}:\right]}^{\mathrm{H}}\left[\widehat{\boldsymbol{H}}_{b^{*}}^{a^{*}}\right]_{\left[n_{r}^{*}:\right]}\right\}\right\} \\
=\operatorname{Tr}\left\{\nu^{2}\left[\boldsymbol{M}_{b^{*}}^{a^{*}}\right]_{\left(n_{r}^{*}, n_{r}^{*}\right)}+\left[\boldsymbol{\Phi}_{b^{*}}^{a^{*}}\right]_{\left(n_{r}^{*}, n_{r}^{*}\right)}\right\},
\end{aligned}
$$

where $\operatorname{Tr}\{\}$ denotes the matrix trace operator, and $\left[\boldsymbol{\Phi}_{b^{*}}^{a^{*}}\right]_{(i, j)} \in \mathbb{C}^{N_{t} \times N_{t}}, \forall i, j \in\left\{1,2, \cdots, N_{r}\right\}$, is the $(i, j)$ th sub-matrix of $\boldsymbol{\Phi}_{b^{*}}^{a^{*}}$ which has a structure similar to (24). Thus, by denoting

$$
\left[\boldsymbol{\Theta}_{b^{*}}^{a^{*}}\right]_{\left(n_{r}^{*}, n_{r}^{*}\right)}=\nu^{2}\left[\boldsymbol{M}_{b^{*}}^{a^{*}}\right]_{\left(n_{r}^{*}, n_{r}^{*}\right)}+\left[\boldsymbol{\Phi}_{b^{*}}^{a^{*}}\right]_{\left(n_{r}^{*}, n_{r}^{*}\right)},
$$

we have

$$
\left|\mathcal{E}\left\{\left[\boldsymbol{H}_{b^{*}}^{a^{*}}\right]_{\left[n_{r}^{*}:\right]}\left[\boldsymbol{V}_{b^{*}}^{a^{*}}\right]_{\left[: n_{r}^{*}\right]}\right\}\right|^{2}=\left(\operatorname{Tr}\left\{\left[\boldsymbol{\Theta}_{b^{*}}^{a^{*}}\right]_{\left(n_{r}^{*}, n_{r}^{*}\right)}\right\}\right)^{2} .
$$

Note that multiplying (29) with $P_{r, b^{*}}^{a^{*}}$ leads to the desired signal power, i.e. the numerator of the SINR expression (15).

As shown in the Appendix, as $N_{t} \rightarrow \infty$, we have

$$
\begin{aligned}
\operatorname{Var}\left\{\left[\boldsymbol{H}_{b^{*}}^{a^{*}}\right]_{\left[n_{r}^{*}:\right]}\left[\boldsymbol{V}_{b^{*}}^{a^{*}}\right]_{\left[: n_{r}^{*}\right]}\right\} \\
=\operatorname{Tr}\left\{\left[\boldsymbol{\Xi}_{b^{*}}^{a^{*}}\right]_{\left(n_{r}^{*}, n_{r}^{*}\right)}\left[\boldsymbol{\Theta}_{b^{*}}^{a^{*}}\right]_{\left(n_{r}^{*}, n_{r}^{*}\right)}\right\} .
\end{aligned}
$$

Additionally, recalling (9) and after some simplifications, we can express $\mathcal{E}\left\{\left|\left[\boldsymbol{H}_{b^{*}}^{a^{*}}\right]_{\left[n_{r}^{*}:\right]}\left[\boldsymbol{V}_{b^{*}}^{a^{*}}\right]_{\left[: n_{r}\right]}\right|^{2}\right\}$ as

$\mathcal{E}\left\{\left|\left[\boldsymbol{H}_{b^{*}}^{a^{*}}\right]_{\left[n_{r}^{*}:\right]}\left[\boldsymbol{V}_{b^{*}}^{a^{*}}\right]_{\left[: n_{r}\right]}\right|^{2}\right\}=\operatorname{Tr}\left\{\left(\nu^{2}\left[\boldsymbol{M}_{b^{*}}^{a^{*}}\right]_{\left(n_{r}, n_{r}\right)}\right.\right.$

$$
\begin{aligned}
& +\left(\left[\boldsymbol{\Phi}_{b^{*}}^{a^{*}}\right]_{\left(n_{r}, n_{r}\right)}+\frac{\sigma_{w}^{2}}{P_{r, b^{*}}^{a^{*}}} \boldsymbol{I}_{N_{t}}+\sum_{a=1}^{A} \frac{P_{r, a^{*}}^{a}}{P_{r, b^{*}}^{a^{*}}} \varsigma^{2}\left[\overline{\boldsymbol{R}}_{\mathrm{r}, r}^{t}\right]_{\left(n_{r}, n_{r}\right)}\right) \\
& \left.\left.\times\left[\boldsymbol{\Omega}_{b^{*}}^{a^{*}}\right]_{\left(n_{r}, n_{r}\right)}\right)\left(\nu^{2}\left[\boldsymbol{M}_{b^{*}}^{a^{*}}\right]_{\left(n_{r}^{*}, n_{r}^{*}\right)}+\varsigma^{2}\left[\overline{\boldsymbol{R}}_{\mathrm{r}, r}^{t}\right]_{\left(n_{r}^{*}, n_{r}^{*}\right)}\right)\right\},
\end{aligned}
$$

asymptotically, where $\left[\boldsymbol{\Omega}_{b^{*}}^{a^{*}}\right]_{\left(n_{r}, n_{r}\right)} \in \mathbb{C}^{N_{t} \times N_{t}}$ is given by

$$
\begin{aligned}
{\left[\boldsymbol{\Omega}_{b^{*}}^{a^{*}}\right]_{\left(n_{r}, n_{r}\right)}=} & \varsigma^{2}\left[\overline{\boldsymbol{R}}_{\mathrm{r}, r}^{t}\right]_{\left(n_{r}, n_{r}\right)}\left(\frac{\sigma_{w}^{2}}{P_{r, b^{*}}^{a^{*}}} \boldsymbol{I}_{N_{t}}+\left[\overline{\boldsymbol{R}}_{\mathrm{r}, r}^{t}\right]_{\left(n_{r}, n_{r}\right)}\right. \\
& \left.+\sum_{a=1}^{A} \frac{P_{r, a^{*}}^{a}}{P_{r, b^{*}}^{a^{*}}} \varsigma^{2}\left[\overline{\boldsymbol{R}}_{\mathrm{r}, r}^{t}\right]_{\left(n_{r}, n_{r}\right)}\right)^{-1}
\end{aligned}
$$

in which $\left[\overline{\boldsymbol{R}}_{\mathrm{r}, r}^{t}\right]_{(i, j)} \in \mathbb{C}^{N_{t} \times N_{t}}, \forall i, j \in\left\{1,2, \cdots, N_{r}\right\}$, represents the $(i, j)$-th sub-matrix of $\overline{\boldsymbol{R}}_{\mathrm{r}, r}^{t}$, which has a structure similar to (24). Similarly, we can express 


$$
\begin{aligned}
& P_{\mathrm{I} \& \mathrm{~N}_{b^{*}, n_{r}}^{a^{*}}}=\sigma_{w}^{2}+P_{r, b^{*}}^{a^{*}} \operatorname{Tr}\left\{\left[\boldsymbol{\Xi}_{b^{*}}^{a^{*}}\right]_{\left(n_{r}^{*}, n_{r}^{*}\right)}\left[\boldsymbol{\Theta}_{b^{*}}^{a^{*}}\right]_{\left(n_{r}^{*}, n_{r}^{*}\right)}\right\}+P_{r, b^{*}}^{a^{*}} \sum_{\substack{n_{r}=1 \\
n_{r} \neq n_{r}^{*}}}^{N_{r}} \operatorname{Tr}\left\{\left(\nu^{2}\left[\boldsymbol{M}_{b^{*}}^{a^{*}}\right]_{\left(n_{r}, n_{r}\right)}+\left(\left[\boldsymbol{\Phi}_{b^{*}}^{a^{*}}\right]_{\left(n_{r}, n_{r}\right)}+\frac{\sigma_{w}^{2}}{P_{r, b^{*}}^{a^{*}}} \boldsymbol{I}_{N_{t}}\right.\right.\right. \\
& \left.\left.\left.+\frac{A \bar{P}_{r}}{P_{r, b^{*}}^{a^{*}}} \varsigma^{2}\left[\overline{\boldsymbol{R}}_{\mathbf{r}, r}^{t}\right]_{\left(n_{r}, n_{r}\right)}\right)\left[\boldsymbol{\Omega}_{b^{*}}^{a^{*}}\right]_{\left(n_{r}, n_{r}\right)}\right)\left(\nu^{2}\left[\boldsymbol{M}_{b^{*}}^{a^{*}}\right]_{\left(n_{r}^{*}, n_{r}^{*}\right)}+\varsigma^{2}\left[\overline{\boldsymbol{R}}_{\mathbf{r}, r}^{t}\right]_{\left(n_{r}^{*}, n_{r}^{*}\right)}\right)\right\}+\bar{P}_{r} \sum_{a=1}^{A} \sum_{n_{r}=1}^{N_{r}} \operatorname{Tr}\left\{\left(\nu^{2}\left[\boldsymbol{M}_{b^{a}}^{a}\right]_{\left(n_{r}, n_{r}\right)}\right.\right. \\
& \left.\left.+\left(\left[\boldsymbol{\Phi}_{b^{a}}^{a}\right]_{\left(n_{r}, n_{r}\right)}+\frac{A \bar{P}_{r}}{P_{r, b^{*}}^{a^{*}}} \varsigma^{2}\left[\overline{\boldsymbol{R}}_{\mathbf{r}, r}^{t}\right]_{\left(n_{r}, n_{r}\right)}+\frac{\sigma_{w}^{2}}{P_{r, b^{*}}^{a^{*}}} \boldsymbol{I}_{N_{t}}\right)\left[\boldsymbol{\Omega}_{b^{a}}^{a}\right]_{\left(n_{r}, n_{r}\right)}\right)\left(\nu^{2}\left[\boldsymbol{M}_{b^{*}}^{a}\right]_{\left(n_{r}^{*}, n_{r}^{*}\right)}+\varsigma^{2}\left[\overline{\boldsymbol{R}}_{\mathrm{r}, r}^{t}\right]_{\left(n_{r}^{*}, n_{r}^{*}\right)}\right)\right\} .
\end{aligned}
$$

$$
\begin{aligned}
& \mathcal{E}\left\{\left|\left[\boldsymbol{H}_{b^{*}}^{a}\right]_{\left[n_{r}^{*}:\right]}\left[\boldsymbol{V}_{b^{a}}^{a}\right]_{\left[: n_{r}\right]}\right|^{2}\right\} \text { asymptotically as } \\
& \mathcal{E}\left\{\left|\left[\boldsymbol{H}_{b^{*}}^{a}\right]_{\left[n_{r}^{*}:\right]}\left[\boldsymbol{V}_{b^{a}}^{a}\right]_{\left[: n_{r}\right]}\right|^{2}\right\}=\operatorname{Tr}\left\{\left(\nu^{2}\left[\boldsymbol{M}_{b^{a}}^{a}\right]_{\left(n_{r}, n_{r}\right)}\right.\right. \\
& \quad+\left(\left[\boldsymbol{\Phi}_{b^{a}}^{a}\right]_{\left(n_{r}, n_{r}\right)}+\sum_{a=1}^{A} \frac{P_{r, a^{*}}^{a} \varsigma^{2}}{P_{r, b^{*}}^{a *}}\left[\overline{\boldsymbol{R}}_{\mathrm{r}, r}^{t}\right]_{\left(n_{r}, n_{r}\right)}+\frac{\sigma_{w}^{2}}{P_{r, b^{*}}^{a^{*}}} \boldsymbol{I}_{N_{t}}\right) \\
& \left.\left.\quad \times\left[\boldsymbol{\Omega}_{b^{a}}^{a}\right]_{\left(n_{r}, n_{r}\right)}\right)\left(\nu^{2}\left[\boldsymbol{M}_{b^{*}}^{a}\right]_{\left(n_{r}^{*}, n_{r}^{*}\right)}+\varsigma^{2}\left[\overline{\boldsymbol{R}}_{\mathrm{r}, r}^{t}\right]_{\left(n_{r}^{*}, n_{r}^{*}\right)}\right)\right\} .
\end{aligned}
$$

Upon substituting (30), (31) and (33) into (16), therefore, we arrive asymptotically at the power of the interference plus noise $P_{\mathrm{I \& N}_{b^{*} n_{n}}^{a^{*}}}$ given in (34) at the top of this page. Furthermore, substituting (29) into (15) leads to the following asymptotic SINR expression

$$
\gamma_{b^{*}, n_{r}}^{a^{*}}=\frac{P_{r, b^{*}}^{a^{*}}\left(\operatorname{Tr}\left\{\left[\Theta_{b^{*}}^{a^{*}}\right]_{\left(n_{r}^{*}, n_{r}^{*}\right)}\right\}\right)^{2}}{P_{\mathrm{I \& N} \mathrm{N}_{b^{*}, n_{r}}^{a^{*}}}} .
$$

Remark 1: Both $\left[\boldsymbol{M}_{b^{a}}^{a}\right]_{\left(n_{r}, n_{r}\right)}$ and $\left[\boldsymbol{M}_{b^{*}}^{a}\right]_{\left(n_{r}^{*}, n_{r}^{*}\right)}$ in (34) are unavailable to aircraft $a^{*}$, since there is no cooperation between the related aircraft. However, both these two terms can be substituted by $\left[\boldsymbol{M}_{b^{*}}^{a^{*}}\right]_{\left(n_{r}^{*}, n_{r}^{*}\right)}$ as a reasonable approximation. The simulation results presented in Section IV will demonstrate that this approximation is sufficiently accurate.

Remark 2: The high velocity of aircraft results in rapidly fluctuating fading. The channel estimator (9) is the most efficient, since its mean square error matches the CramérRao lower bound. However, by the time the transmitter transmits the precoded signal based on this channel estimate, the real channel has changed. This mobility-induced channel estimation 'error' will degrade the achievable performance. An effective approach to mitigate this performance degradation owing to channel estimation errors is to adopt robust transmit precoding. The design of robust precoding is beyond the scope of this paper. Some highly effective robust precoding designs can be found in [36], [37].

Remark 3: As our derivation does not impose any specific geometric structure on the antenna array, our results and therefore our proposed physical-layer transmission scheme is applicable to systems equipped with uniformly spaced linear arrays (ULAs), uniformly spaced rectangular arrays (URAs), or any other generic antenna arrays.

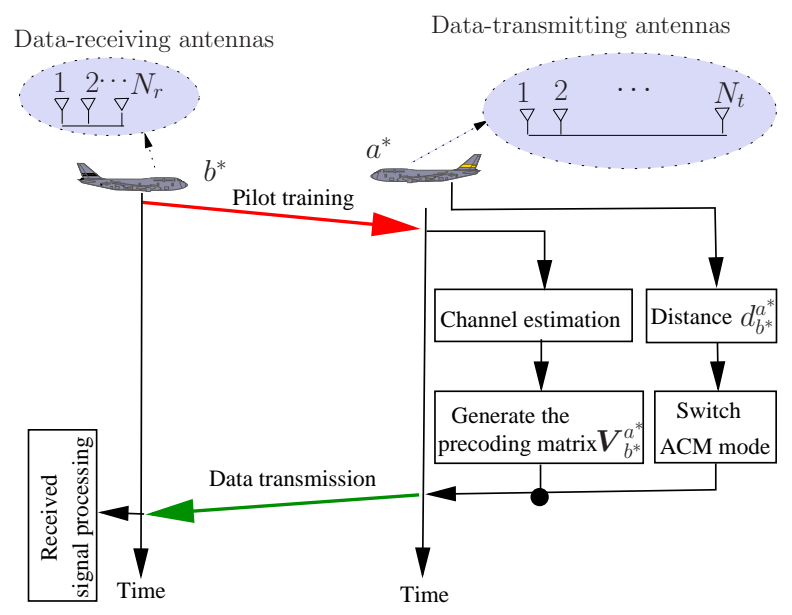

Fig. 2. Schematic of the proposed distance-based adaptive coding and modulation scheme.

\section{B. Distance-based ACM scheme}

The proposed distance-based ACM scheme is illustrated in Fig. 2. Our scheme explicitly uses the distance $d_{b^{*}}^{a^{*}}$ between aircraft $a^{*}$ and aircraft $b^{*}$ as the switching metric to adapt the modulation mode and code rate. Similar to the conventional ACM scheme, our distance-based ACM also consists of the set of $K$ ACM modes, but its switching thresholds comprise the $K$ distance thresholds $\left\{d_{k}\right\}_{k=1}^{K}$. An example of this distancebased ACM scheme using $K=7$ is given in Table I, where the SE is calculated as: $\log _{2}$ (modulation order) $\times$ code rate $\times\left[\left(N-N_{\mathrm{cp}}\right) / N\right]$, and the data rate per DRA is

TABLE I

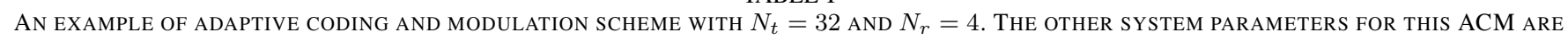
LISTED IN TABLE III.

\begin{tabular}{|c|c|c|c|c|c|c|}
\hline Mode $k$ & Modulation & Code rate & $\begin{array}{c}\text { Spectral efficiency } \\
(\mathrm{bps} / \mathrm{Hz})\end{array}$ & $\begin{array}{c}\text { Switching threshold } \\
d_{k}(\mathrm{~km})\end{array}$ & $\begin{array}{c}\text { Data rate per receive } \\
\text { antenna }(\mathrm{Mbps})\end{array}$ & $\begin{array}{c}\text { Total data rate } \\
(\mathrm{Mbps})\end{array}$ \\
\hline 1 & BPSK & 0.488 & 0.459 & 500 & 2.754 & 11.016 \\
\hline 2 & QPSK & 0.533 & 1.000 & 350 & 6.000 & 24.000 \\
\hline 3 & QPSK & 0.706 & 1.322 & 200 & 7.932 & 31.728 \\
\hline 4 & 8-QAM & 0.642 & 1.809 & 110 & 10.854 & 43.416 \\
\hline 5 & 8-QAM & 0.780 & 2.194 & 40 & 13.164 & 52.656 \\
\hline 6 & 16-QAM & 0.731 & 2.747 & 25 & 16.482 & 65.928 \\
\hline 7 & 16-QAM & 0.853 & 3.197 & 5.56 & 19.182 & 76.728 \\
\hline
\end{tabular}




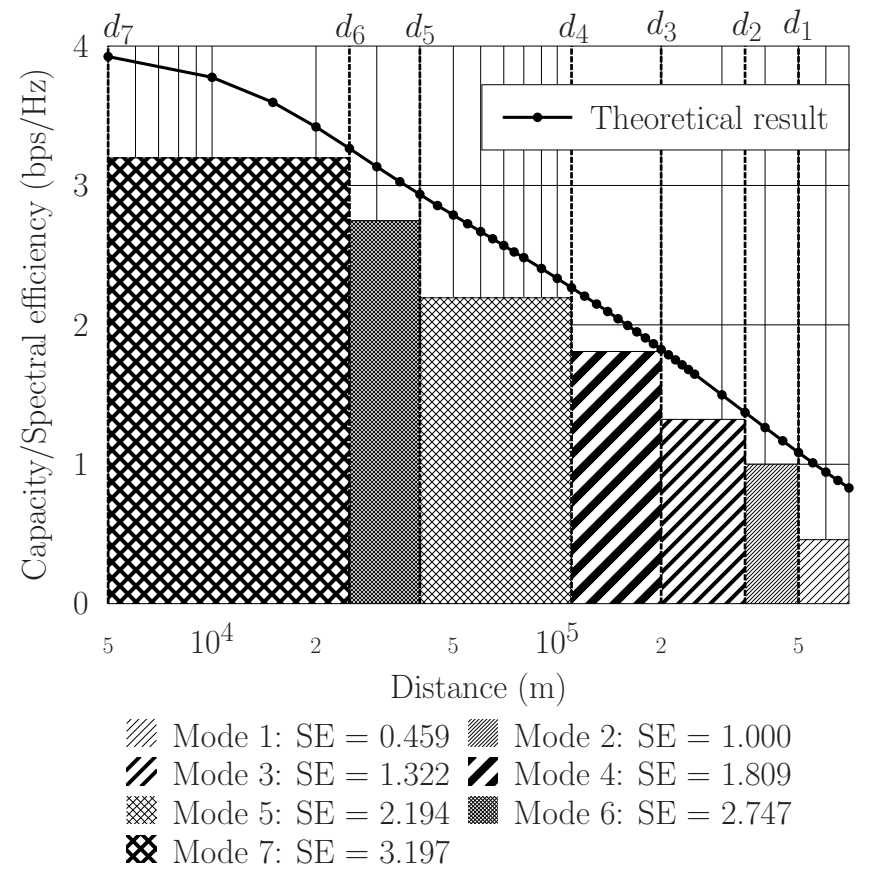

(a) $N_{t}=32, N_{r}=4$

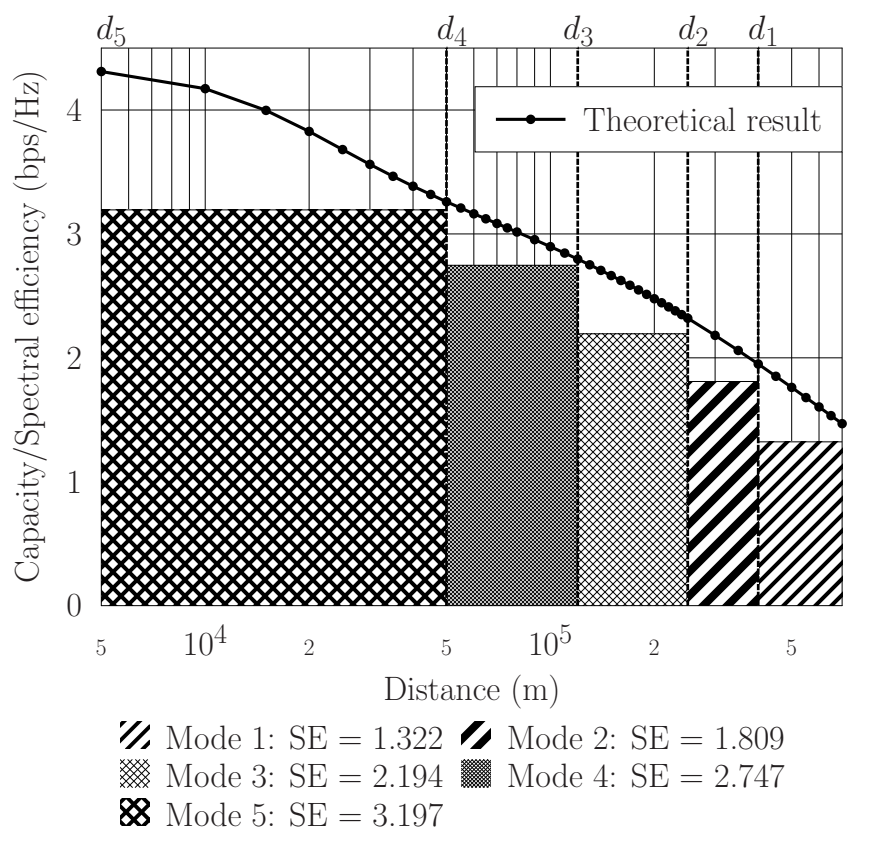

(b) $N_{t}=64, N_{r}=4$

Fig. 3. Illustration of how to obtain the desired distance thresholds for the proposed distance-based ACM scheme, using the examples of Tables I and II.

calculated as: spectral efficiency $\times B_{\text {total }}$, in which $B_{\text {total }}$ is the bandwidth available, while the total data rate is given by: data rate per DRA $\times N_{r}$. The modulation schemes and code rates are adopted from the design of VersaFEC [38], which covers a family of 12 short-block LDPC code rates with the matched modulation schemes. VersaFEC is specifically designed for low latency and ACM applications. The operations of this distance-based ACM are now given below.

1) Aircraft $a^{*}$ estimates the channel $\boldsymbol{H}_{b^{*}}^{a^{*}}$ based on the pilots transmitted by aircraft $b^{*}$, as detailed in Section II-A.

2) Aircraft $a^{*}$ calculates the TPC matrix $V_{b^{*}}^{a^{*}}$ according to (11).

3) Aircraft $a^{*}$ selects an ACM mode to transmit the data according to

$$
\text { If } d_{k} \leq d_{b^{*}}^{a^{*}}<d_{k-1}: \text { choose mode } k,
$$

where $k \in\{1,2, \cdots, K\}$, and we assume $d_{0}=D_{\max }$. When $d_{b^{*}}^{a^{*}} \geq D_{\max }$, there exists no available communication link, since the two aircraft are beyond each others' communication range. Since the minimum flight-safety separation must be obeyed, we do not consider the senario of $d_{b^{*}}^{a^{*}} \leq D_{\min }$.

The switching distance threshold for each ACM mode is determined based on the achievable rate per DRA as a function of distance. Specifically, the theoretically achievable rate per
DRA as a function of distance is calculated using (15). The distance thresholds $\left\{d_{k}\right\}_{k=1}^{K}$ are chosen so that the SE of mode $k$ is lower than the theoretically achievable rate per DRA in the distance range of $\left[d_{k}, d_{k-1}\right]$ to ensure successful transmission. Fig. 3(a) illustrates how the 7 distance thresholds are designed for the example provided in Table I. For this example, $N_{t}=32, N_{r}=4$, and the total system bandwidth is $B_{\text {total }}=6 \mathrm{MHz}$ which is reused by every aircraft in the system. The theoretically achievable rate per DRA as a function of distance is depicted as the dot-marked solid curve in Fig. 3(a). By designing the 7 distance thresholds $d_{k}$ for $1 \leq k \leq 7$ to ensure that the SE of mode $k$ is lower than the theoretically achievable rate in the distance range $\left[d_{k}, d_{k-1}\right]$, we obtain the 7 desired distance thresholds for this ACM example, which are indicated in Fig. 3(a) as well as listed in Table I. The designed 7 ACM modes are: mode 1 having the SE of $0.459 \mathrm{bps} / \mathrm{Hz}$, mode 2 with the SE $1.000 \mathrm{bps} / \mathrm{Hz}$, mode 3 with the SE $1.322 \mathrm{bps} / \mathrm{Hz}$, mode 4 with the SE 1.809 $\mathrm{bps} / \mathrm{Hz}$, mode 5 with the SE $2.194 \mathrm{bps} / \mathrm{Hz}$, mode 6 with the $2.747 \mathrm{bps} / \mathrm{Hz}$, and mode 7 with the SE $3.197 \mathrm{bps} / \mathrm{Hz}$.

We also provide another design example of the ACM scheme for $K=5$ modes. For this example, we have $N_{t}=64$ and $N_{r}=4$, while the other system parameters are the same as the ACM scheme listed in Table I. As shown in Fig. 3(b) and Table II, the five ACM modes have the SEs

TABLE II

AN EXAMPLE OF ADAPTIVE CODING AND MODULATION SCHEME WITH $N_{t}=64$ AND $N_{r}=4$. THE OTHER SYSTEM PARAMETERS FOR THIS ACM ARE LISTED IN TABLE III.

\begin{tabular}{|c|c|c|c|c|c|c|}
\hline Mode $k$ & Modulation & Code rate & $\begin{array}{c}\text { Spectral efficiency } \\
(\mathrm{bps} / \mathrm{Hz})\end{array}$ & $\begin{array}{c}\text { Switching threshold } \\
d_{k}(\mathrm{~km})\end{array}$ & $\begin{array}{c}\text { Data rate per receive } \\
\text { antenna }(\mathrm{Mbps})\end{array}$ & $\begin{array}{c}\text { Total data rate } \\
(\mathrm{Mbps})\end{array}$ \\
\hline 1 & QPSK & 0.706 & 1.322 & 400 & 7.932 & 31.728 \\
\hline 2 & 8-QAM & 0.642 & 1.809 & 250 & 10.854 & 43.416 \\
\hline 3 & 8-QAM & 0.780 & 2.194 & 120 & 13.164 & 52.656 \\
\hline 4 & 16-QAM & 0.731 & 2.747 & 50 & 16.482 & 65.928 \\
\hline 5 & 16-QAM & 0.853 & 3.197 & 5.56 & 19.182 & 76.728 \\
\hline
\end{tabular}


of $1.322 \mathrm{bps} / \mathrm{Hz}, 1.809 \mathrm{bps} / \mathrm{Hz}, 2.194 \mathrm{bps} / \mathrm{Hz}, 2.747 \mathrm{bps} / \mathrm{Hz}$ and $3.197 \mathrm{bps} / \mathrm{Hz}$, respectively, while the corresponding switching thresholds are $400 \mathrm{~km}, 250 \mathrm{~km}, 120 \mathrm{~km}, 50 \mathrm{~km}$, and $5.56 \mathrm{~km}$, respectively. By comparing Table II to Table I, it can be seen that employing a larger number of transmit antennas enables an ACM mode to operate over a larger range of distances, or it allows the system to transmit at a higher SE over a given communication distance. This makes sense because a well-known MIMO property is that employing more transmit antennas can mitigate the interference more effectively.

Remark 4: It is worth recapping that the conventional instantaneous SNR-based ACM is unsuitable for aeronautical communication applications, because the speed of aircraft is ultra high, which results in rapidly changing of largescale fading and consequently very unreliable estimate of the instantaneous SNR as well as leads to frequently switching among the modes. Using erroneous instantaneous SNR estimates to frequently switch modes will cause frequent unsuccessful transmissions. By contrast, the proposed ACM scheme switches its mode based on the distance, which is readily available to the transmitting aircraft, since every jumbo jet has a radar and is equipped with GPS. It can be seen that this distance-based ACM scheme is particularly suitable for aeronautical communication applications. Furthermore, it is worth emphasizing that using the distance as the switching metric is theoretically well justified, because for the aeronautical communication channel the achievable capacity is mainly dependent on the distance, as we have analytically derived in Subsection III-A. Note that owing to the high velocity of the aircraft, no physical layer transmission scheme can guarantee successful transmission for every transmission slot. Other higher-layer measures, such as Automatic-RepeatreQuest (ARQ) [39], [40], can be employed for enhancing reliable communication among aircraft. Discussing these higherlayer techniques is beyond the scope of this paper.

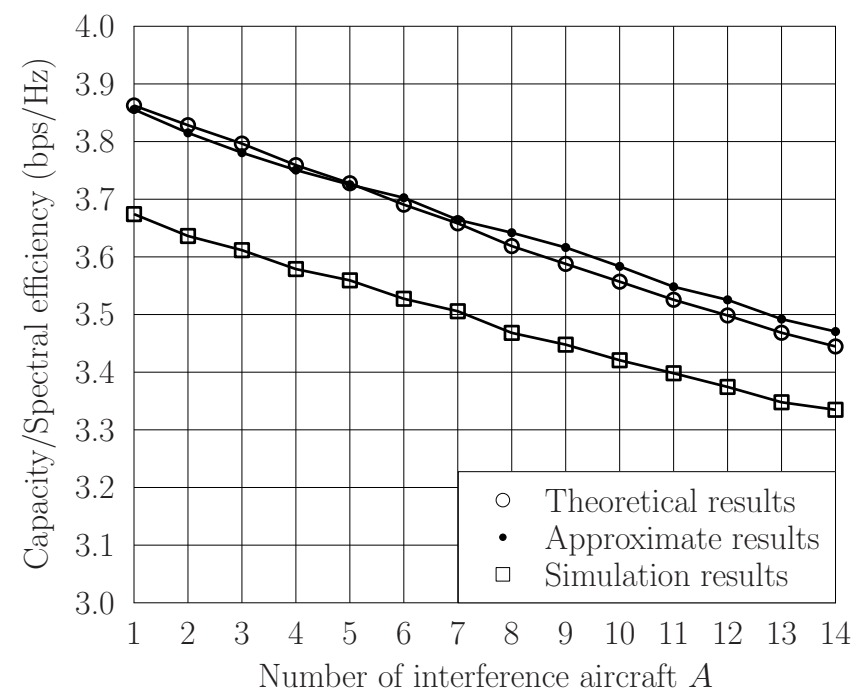

(a) Throughput
TABLE III

DEFAULT PARAMETERS USED IN THE SIMULATED AERONAUTICAL COMMUNICATION SYSTEM

\begin{tabular}{l|l}
\hline \hline \multicolumn{2}{l}{ System parameters for ACM } \\
\hline Number of interference aircraft $A$ & 4 \\
\hline Number of DRAs $N_{r}$ & 4 \\
\hline Number of DTAs $N_{t}$ & 32 \\
\hline Transmit power per antennas $P_{t}$ & 1 watt \\
\hline Number of total subcarriers $N$ & 512 \\
\hline Number of CPs $N_{\mathrm{cp}}$ & 32 \\
\hline Rician factor $K_{\text {Rice }}$ & 5 \\
\hline Bandwidth $B_{\text {total }}$ & $6 \mathrm{MHz}$ \\
\hline Frequency of centre subcarrier & $5 \mathrm{GHz}$ \\
\hline \hline Other system parameters & 0.1 \\
\hline Correlation factor between antennas $\rho$ & $4 \mathrm{~dB}$ \\
\hline Noise figure at receiver $F$ & $10 \mathrm{~km}$ \\
\hline Distance between communicating aircraft $a^{*}$ and $b^{*} d_{b^{*}}^{a^{*}}$ & $740 \mathrm{~km}$ \\
\hline Maximum communication distance $D_{\max }$ &
\end{tabular}

\section{Simulation STUdY}

The default values of the parameters used for our simulated aeronautical communication system are summarized in Table III. Unless otherwise specified, these default values are used. The number of DRAs is much lower than the number of DTAs in order to ensure that the DRAs' signals remain uncorrelated to avoid the interference among the antennas at the receiver. The deterministic part of the Rician channel, which satisfies $\operatorname{Tr}\left\{\boldsymbol{H}_{\mathrm{d}, b}^{a} \boldsymbol{H}_{\mathrm{d}, b}^{a, \mathrm{H}}\right\}=N_{t} N_{r}$, is generated in every Monte-Carlo simulation. The scattering component of the Rician channel $\boldsymbol{H}_{\mathrm{r}, b}^{a} \in \mathbb{C}^{N_{r} \times N_{t}}$ is generated according to

$$
\boldsymbol{H}_{\mathrm{r}, b}^{a}=\boldsymbol{R}_{b} \boldsymbol{G} \boldsymbol{R}^{a}
$$

where $\boldsymbol{R}_{b}=\boldsymbol{I}_{N_{r}}$, since the DRAs are uncorrelated, but the $m$ th-row and $n$ th-column element of the correlation matrix of $\boldsymbol{R}^{a}$, denoted by $\left[\boldsymbol{R}^{a}\right]_{[m, n]}$, is generated according to [41], [42]

$$
\left[\boldsymbol{R}^{a}\right]_{[m, n]}=\left(\left[\boldsymbol{R}^{a}\right]_{[n, m[}\right)^{\ddagger}=(t \rho)^{|m-n|},
$$

in which ( $)^{\ddagger}$ denotes conjugate operation, and $t \sim \mathcal{C N}(0,1)$ is the magnitude of the correlation coefficient, which is deter-

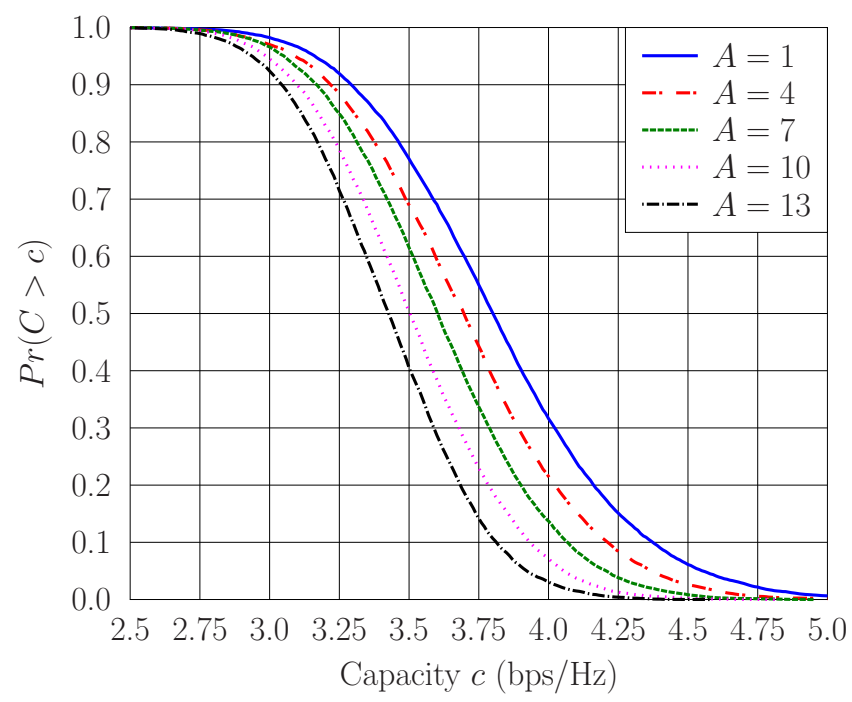

(b) $\mathrm{CCDF}$

Fig. 4. (a) The achievable throughput per DRA as a function of the number of interfering aircraft $A$, and (b) The CCDFs of the simulated throughputs per DRA for different numbers of interfering aircraft. The distances between the interfering aircraft and the desired receiving aircraft are uniformly distributed within the range of $\left[d_{b^{*}}^{a^{*}}, D_{\max }\right]$. The rest of the parameters are specified in Table III. 
mined by the antenna element spacing [42]. The antenna array correlation matrix model (38) is derived based on the ULA, and this implies that we adopt the ULA in our simulation study. However, it is worth recalling Remark 3 stating that our scheme is not restricted to the ULA. In the investigation of the achievable throughput, 'Theoretical' is the throughput calculated using (17) relying on the perfect knowledge of $\left[\boldsymbol{M}_{b^{a}}^{a}\right]_{\left(n_{r}, n_{r}\right)}$ and $\left[\boldsymbol{M}_{b^{*}}^{a}\right]_{\left(n_{r}^{*}, n_{r}^{*}\right)}$ in (34), and 'Approximate' is the throughput calculated using (15) with both $\left[\boldsymbol{M}_{b^{a}}^{a}\right]_{\left(n_{r}, n_{r}\right)}$ and $\left[\boldsymbol{M}_{b^{*}}^{a}\right]_{\left(n_{r}^{*}, n_{r}^{*}\right)}$ substituted by $\left[\boldsymbol{M}_{b^{*}}^{a^{*}}\right]_{\left(n_{r}^{*}, n_{r}^{*}\right)}$ in (34), while 'Simulation' is the Monte-Carlo simulation result.

\section{A. Results}

Fig. 4(a) investigates the achievable throughput per DRA as a function of the number of interfering aircraft $A$, where the

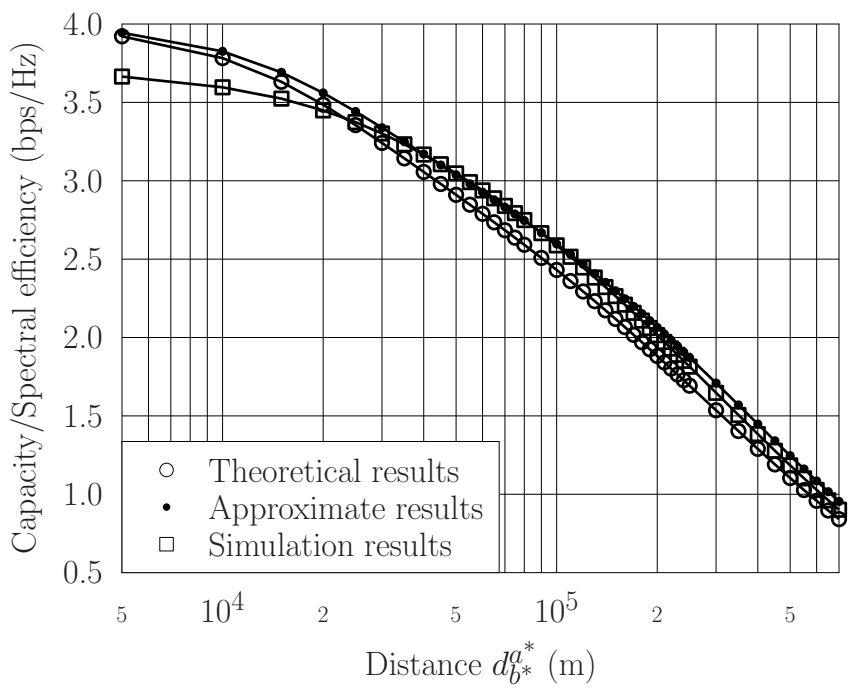

(a) Throughput
'Approximate results' match closely the 'Theoretical results', confirming that $\left[\boldsymbol{M}_{b^{*}}^{a^{*}}\right]_{\left(n^{*}, n^{*}\right)}$ is an accurate approximation of both the unknown $\left[\boldsymbol{M}_{b^{a}}^{a}\right]_{\left(n_{r}, n_{r}\right)}$ and $\left[\boldsymbol{M}_{b^{*}}^{a}\right]_{\left(n_{r}^{*}, n_{r}^{*}\right)}$. As expected, the achievable throughput degrades as the number of interfering aircraft increases. Also the theoretical throughput is about $0.2 \mathrm{bps} / \mathrm{Hz}$ higher than the simulated throughput. This is because the theoretical throughput is obtained by using the asymptotic interference plus noise power as $N_{t} \rightarrow \infty$ and, therefore, it represents the asymptotic upper bound of the achievable throughput. The complementary cumulative distribution functions (CCDFs) of the simulated throughput recorded for different numbers of interfering aircraft $A$ are shown in Fig. 4(b), which characterizes the probability of the achievable throughput above a given value.

Fig. 5(a) portrays the achievable throughput per DRA as

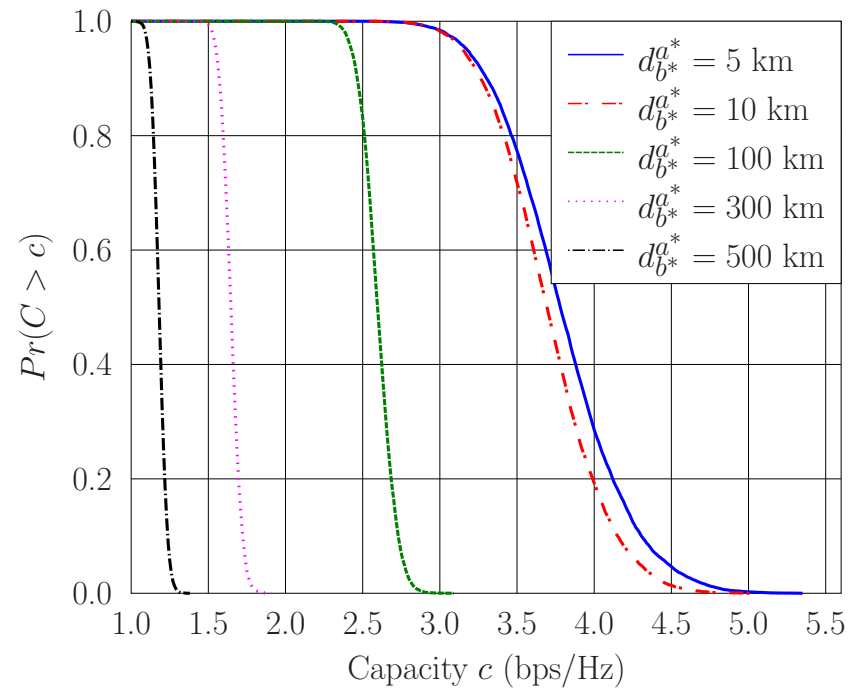

(b) $\mathrm{CCDF}$

Fig. 5. (a) The achievable throughput per DRA as a function of the distance $d_{b^{*}}^{a^{*}}$ between the desired communicating aircraft $a^{*}$ and $b^{*}$, and (b) The CCDFs of the simulated throughputs per DRA for different $d_{b^{*}}^{a^{*}}$. The distances between the interfering aircraft and the desired receiving aircraft are uniformly distributed within the range of $\left[d_{b^{*}}^{a^{*}}, D_{\max }\right]$. The rest of the parameters are specified in Table III.

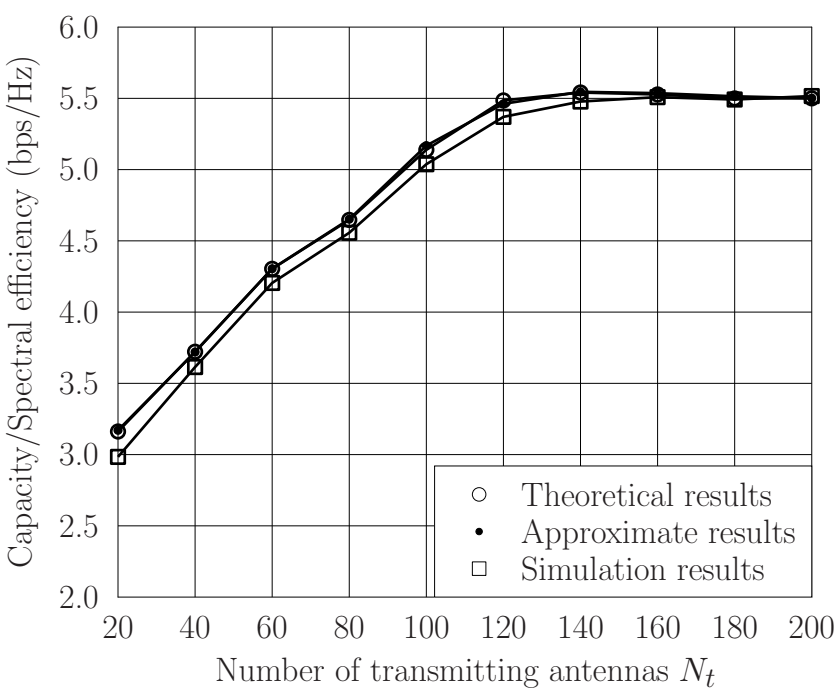

(a) Throughput

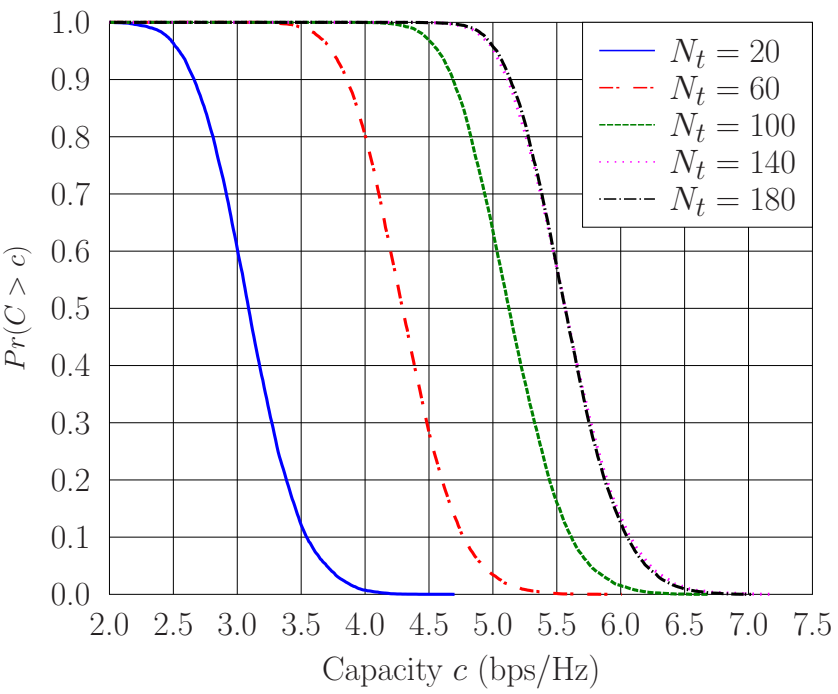

(b) $\mathrm{CCDF}$

Fig. 6. (a) The achievable throughput performance per DRA as a function of the number of DTAs $N_{t}$, and (b) The CCDFs of the simulated throughputs per DRA for different numbers of DTAs $N_{t}$. The distances between the interfering aircraft and the desired receiving aircraft are uniformly distributed within the range of $\left[d_{b^{*}}^{a^{*}}, D_{\max }\right]$. The rest of the parameters are specified in Table III. 
a function of the distance $d_{b^{*}}^{a^{*}}$ between the desired pair of communicating aircraft, while the CCDFs of the simulated throughput recorded for different values of $d_{b^{*}}^{a^{*}}$ are depicted in Fig. 5(b). As expected, the achievable throughput degrades upon increasing the communication distance. Observe that the performance gap between the theoretical curve and the simulation curve at the point of $d_{b^{*}}^{a^{*}}=10 \mathrm{~km}$ is also around $0.2 \mathrm{bps} / \mathrm{Hz}$, which agrees with the results of Fig. 4(a).

The impact of the number of DTAs on the achievable throughput is investigated in Fig. 6. Specifically, Fig. 6(a) shows the throughput per DRA as a function of the number of DTAs $N_{t}$, while Fig. 6(b) depicts the CCDFs of the simulated throughputs per DRA for different numbers of DTAs $N_{t}$. Observe that the achievable throughput increases as $N_{t}$ increases. Moreover, when the number of DTAs increase to

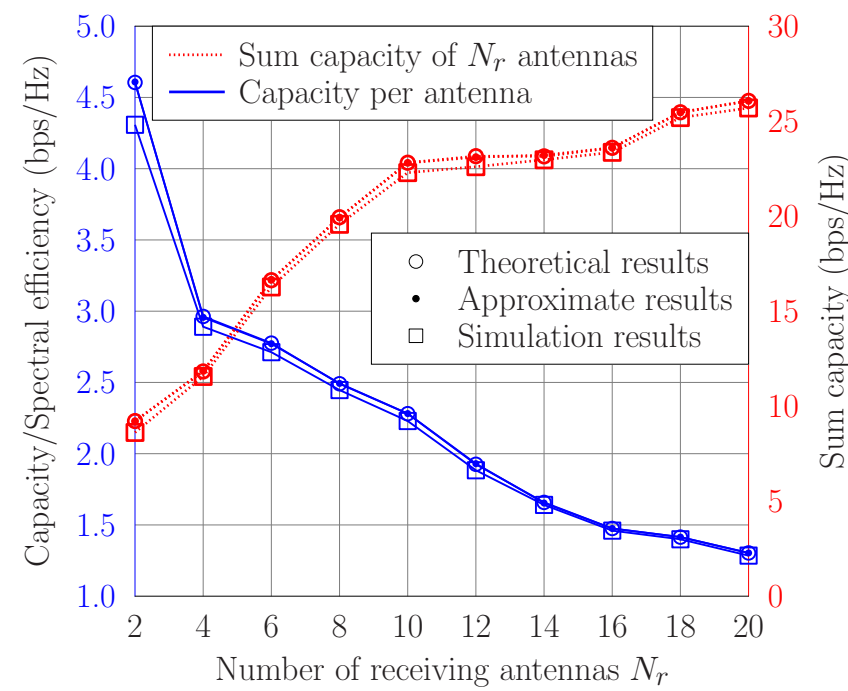

(a) Throughput
$N_{t} \geq 120$, the achievable throughput saturates, as seen from Fig. 6(a). Similarly, the CCDFs recorded for $N_{t}=140$ and $N_{t}=180$ are indistinguishable, as clearly seen from Fig. 6(b). The implication is that the asymptotic performance is reached for $N_{t} \geq 120$.

Next the impact of the number of DRAs on the achievable throughput is studied in Fig. 7. In particular, Fig. 7(a) portrays the achievable throughputs as a functions of $N_{r}$, where the left $y$-axis labels the achievable throughput per DRA and the right $y$-axis indicates the sum rate of the $N_{r}$ DRAs. As expected, the achievable sum rate increases with $N_{r}$. However, the increase in the sum rate is not proportional to the increase of $N_{r}$. In fact, it is clearly seen from Fig. 7(a) that the achievable throughput per DRA is reduced with the increase of $N_{r}$. The reason for this trend is because the inter-antenna interference

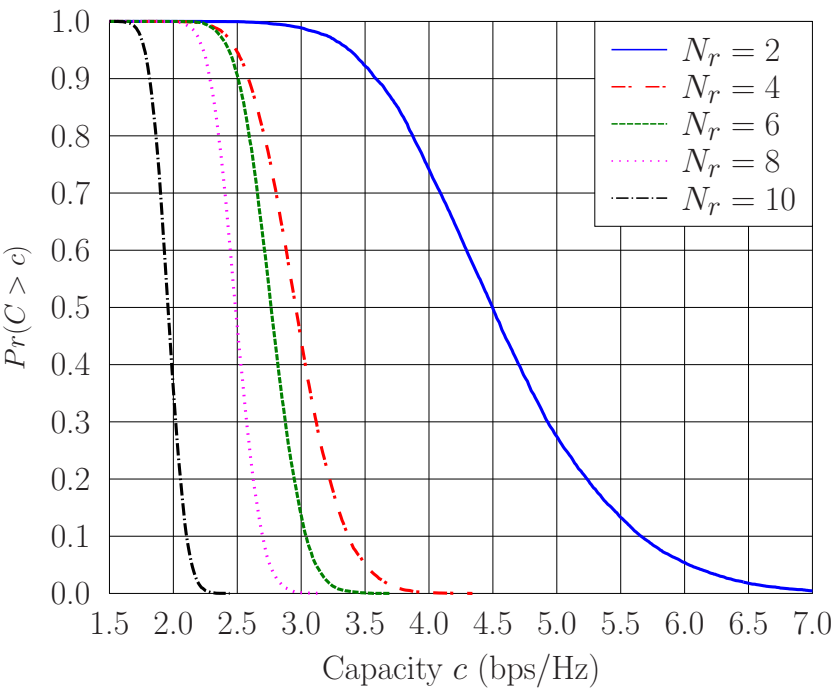

(b) $\mathrm{CCDF}$

Fig. 7. (a) The achievable throughput as a function of the number of DRAs $N_{r}$, and (b) The CCDFs of the simulated throughputs per DRA for different numbers of DRAs $N_{r}$. The distances between the interfering aircraft and the desired receiving aircraft are uniformly distributed within the range of $\left[d_{b^{*}}^{a^{*}}, D_{\max }\right]$. The rest of the parameters are specified in Table III.

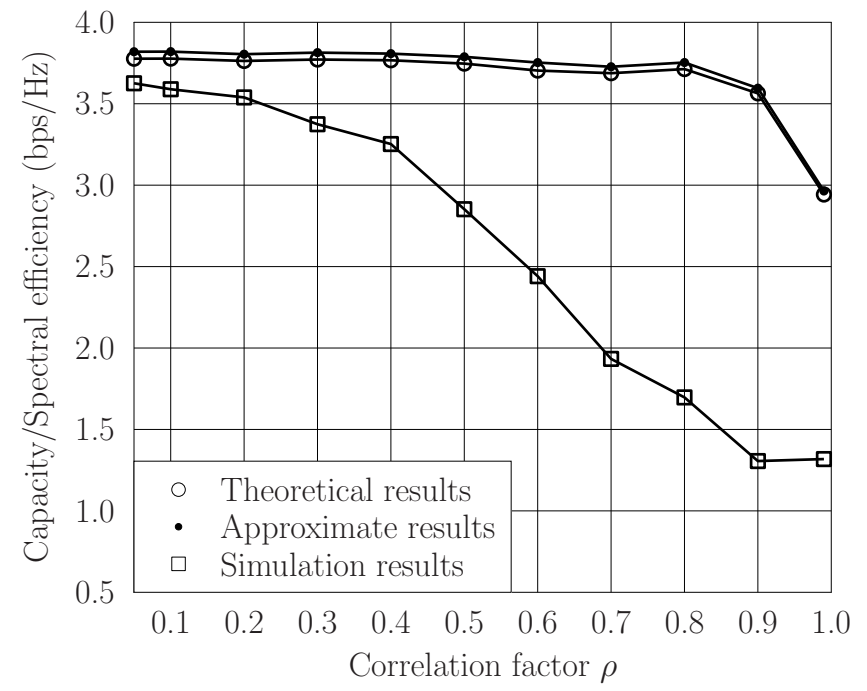

(a) Throughput

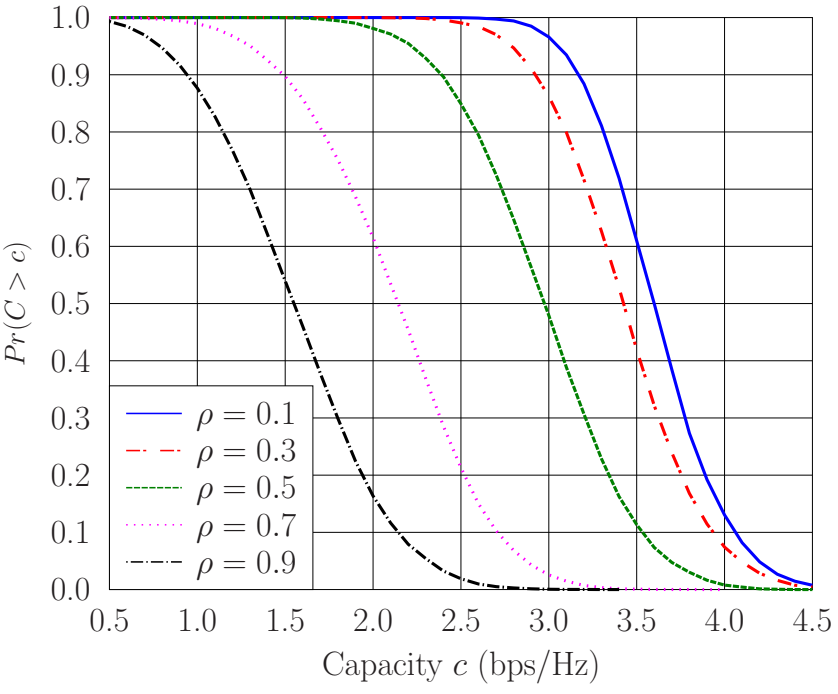

(b) $\mathrm{CCDF}$

Fig. 8. (a) The achievable throughput per DRA as a function of the correlation factor of DTAs $\rho$, and (b) The CCDFs of the simulated throughputs per DRA for different values of $\rho$. The distances between the interfering aircraft and the desired receiving aircraft are uniformly distributed within the range of $\left[d_{b^{*}}^{a^{*}}, D_{\max }\right]$. The rest of the parameters are specified in Table III. 


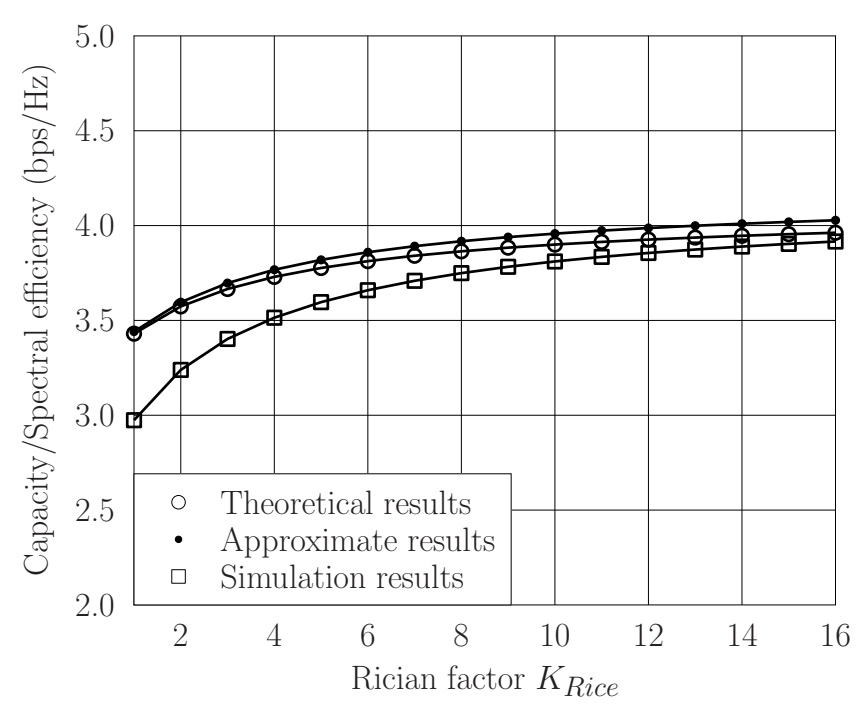

(a) Throughput

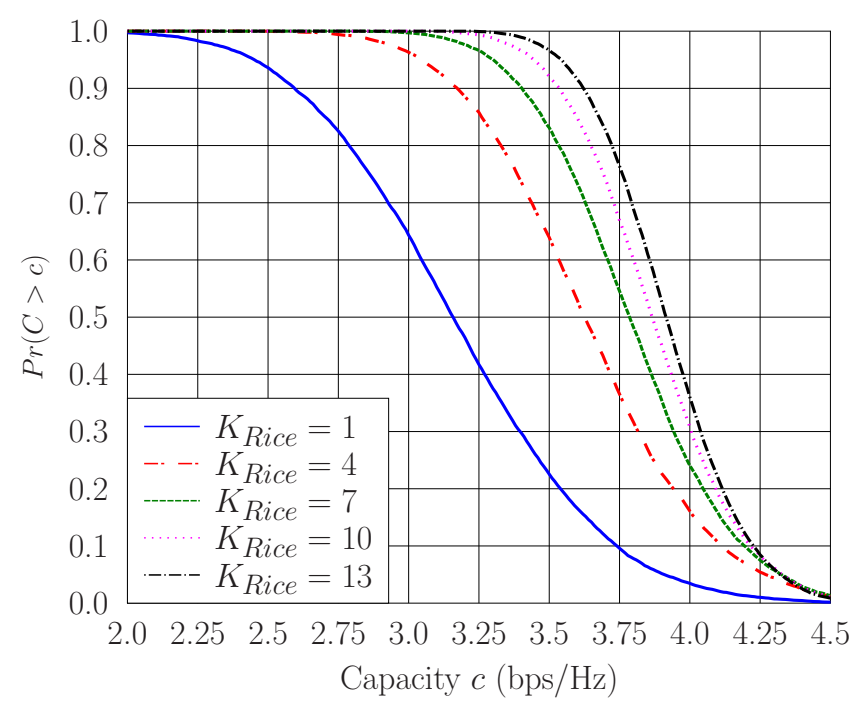

(b) $\mathrm{CCDF}$

Fig. 9. (a) The achievable throughput per DRA as a function of the Rician factor $K_{\text {Rice }}$, and (b) The CCDFs of the simulated throughputs per DRA for different values of $K_{\text {Rice }}$. The distances between the interfering aircraft and the desired receiving aircraft are uniformly distributed within the range of $\left[d_{b^{*}}^{a^{*}}, D_{\max }\right]$. The rest of the parameters are specified in Table III.

increases with the increase of $N_{r}$, as seen in the third and fourth terms of (34). The CCDFs of the simulated throughputs per DRA are illustrated in Fig. 7(b) for different $N_{r}$, which agree with the curve of the simulated capacity per DRA shown in Fig. 7(a), namely the achievable throughput per DRA is lower for larger number of DRAs.

Fig. 8 depicts the impact of the correlation factor $\rho$ of DTAs on the achievable throughput per DRA. It can be seen from Fig. 8(a) that strong signal correlation between DTAs will reduce the achievable throughput, as expected. This degradation is particularly serious in the Monte-Carlo simulation results, i.e. in practice, but less notable for the asymptotic theoretical upper bound. Note that there is a large gap between the theoretical upper bound and the Monte-Carlo simulation results when $\rho \geq 0.4$, indicating that the asymptotic interference plus noise power, which is a lower bound of the true interference plus noise power, is no longer sufficiently tight. The CCDFs of the simulated throughputs per DRA for different values of $\rho$ are shown in Fig. 8(b), which statistically validates the curve of the simulated throughput per DRA given in Fig. 8(a).

The impact of the Rician factor $K_{\text {Rice }}$ on the achievable throughput is shown in Fig. 9. Specifically, Fig. 9(a) depicts the achievable throughputs per DRA as a functions of the Rician factor $K_{\text {Rice }}$, while the CCDFs of the simulated throughput per DRA recorded for different values of $K_{\text {Rice }}$ are given in Fig. 9(b). The results of Fig. 9 clearly show that a higher Rician factor leads to a higher throughput. Observing the channel model (2), we can see that a higher $K_{\text {Rice }}$ results in a larger deterministic or LOS component, which is beneficial for the achievable performance.

\section{B. Discussions}

In the above extensive simulation study, we have carefully investigated how the number of interfering aircraft $A$, the number of DTAs $N_{t}$, the number of DRAs $N_{r}$, the distance $d_{b^{*}}^{a^{*}}$ between the desired pair of communicating aircraft, the correlation factor $\rho$ between antennas, and the Rician factor $K_{\text {Rice }}$ of the aeronautical communication channel impact on the achievable system performance of our distance-based ACM and large-scale antenna array aided AANET. Based on the simulation results, we can draw the following observations.

The distance between the desired pair of communicating aircraft and the correlation factor of DTAs have adverse effects on the achievable system performance. Increasing $d_{b^{*}}^{a^{*}}$ and/or $\rho$ reduces the achievable transmission rate. On the other hand, increasing the number of DTAs, the number of DRAs, and/or the Rician factor is beneficial for the achievable system performance. Specicially, increasing $N_{t}$ and/or $K_{\text {Rice }}$ lead to higher transmission rate, while increasing $N_{r}$ also increases the total transmission rate, although the achieved throughput per DRA is reduced with the increase of $N_{r}$.

Most importantly, our extensive simulation results have validated the design presented in Section III and provide the evidence that our design is capable of supporting the future Internet above the clouds. For example, let us consider the AANET for airborne commercial Internet access having a $5 \mathrm{GHz}$ carrier frequency and a $6 \mathrm{MHz}$ bandwidth, which is spatially shared by $A=14$ other aircraft within the effective communication zone covered by the AANET. When the distance between the desired pair of communicating aircraft is $d_{b^{*}}^{a^{*}}=10 \mathrm{~km}$, our design is capable of offering a total data rate of $79 \mathrm{Mbps}$, as seen from Fig. 4. In the senario of $A=4$ and $d_{b^{*}}^{a^{*}}=70 \mathrm{~km}$, our design is capable of providing a total data rate of $60 \mathrm{Mbps}$, as observed from Fig. 5.

\section{CONCLUSIONS}

A large-scale antenna array aided and novel distance-based ACM scheme has been proposed for aeronautical communications. Unlike the terrestrial instantaneous-SNR based ACM design, which is unsuitable for aeronautical communication applications, the proposed distance-based ACM scheme switches 


$$
\begin{aligned}
& \operatorname{Var}\left\{\left[\boldsymbol{H}_{b^{*}}^{a^{*}}\right]_{\left[n_{r}^{*}:\right]}\left[\boldsymbol{V}_{b^{*}}^{a^{*}}\right]_{\left[: n_{r}^{*}\right]}\right\}=\mathcal{E}\left\{\left|\left(\left[\widehat{\boldsymbol{H}}_{b^{*}}^{a^{*}}\right]_{\left[n_{r}^{*}:\right]}+\left[\widetilde{\boldsymbol{H}}_{b^{*}}^{a^{*}}\right]_{\left[n_{r}^{*}:\right]}\right)\left[\widehat{\boldsymbol{H}}_{b^{*}}^{a^{*}}\right]_{\left[n_{r}^{*}:\right]}^{\mathrm{H}}-\operatorname{Tr}\left\{\left[\boldsymbol{\Theta}_{b^{*}}^{a^{*}}\right]_{\left(n_{r}^{*}, n_{r}^{*}\right)}\right\}\right|^{2}\right\} \\
& \quad \approx \mathcal{E}\left\{\mid\left[\widetilde{\boldsymbol{H}}_{b^{*}}^{a^{*}}\right]_{\left[n_{r}^{*}:\right]}\left[\widehat{\boldsymbol{H}}_{b^{*}}^{a^{*}}\right]_{\left[n_{r}^{*}:\right]}^{\mathrm{H}}\right\}=\mathcal{E}\left\{\left[\widetilde{\boldsymbol{H}}_{b^{*}}^{a^{*}}\right]_{\left[n_{r}^{*}:\right]}\left[\widehat{\boldsymbol{H}}_{b^{*}}^{a^{*}}\right]_{\left[n_{r}^{*}:\right]}^{\mathrm{H}}\left[\widehat{\boldsymbol{H}}_{b^{*}}^{a^{*}}\right]_{\left[n_{r}^{*}:\right]}\left[\widetilde{\boldsymbol{H}}_{b^{*}}^{a^{*}}\right]_{\left[n_{r}^{*}:\right]}^{\mathrm{H}}\right\} \\
& \quad=\mathcal{E}\left\{\left[\widetilde{\boldsymbol{H}}_{b^{*}}^{a^{*}}\right]_{\left[n_{r}^{*}:\right]} \mathcal{E}\left\{\left[\widehat{\boldsymbol{H}}_{b^{*}}^{a^{*}}\right]_{\left[n_{r}^{*}:\right]}^{\mathrm{H}}\left[\widehat{\boldsymbol{H}}_{b^{*}}^{a^{*}}\right]_{\left[n_{r}^{*}:\right]}\right\}\left[\widetilde{\boldsymbol{H}}_{b^{*}}^{a^{*}}\right]_{\left[n_{r}^{*}:\right]}^{\mathrm{H}}\right\}=\mathcal{E}\left\{\left[\widetilde{\boldsymbol{H}}_{b^{*}}^{a^{*}}\right]_{\left[n_{r}^{*}:\right]}\left[\boldsymbol{\Theta}_{b^{*}}^{a^{*}}\right]_{\left(n_{r}^{*}, n_{r}^{*}\right)}\left[\widetilde{\boldsymbol{H}}_{b^{*}}^{a^{*}}\right]_{\left[n_{r}^{*}:\right]}^{\mathrm{H}}\right\} \\
& \quad=\operatorname{Tr}\left\{\left[\boldsymbol{\Xi}_{b^{*}}^{a^{*}}\right]_{\left(n_{r}^{*}, n_{r}^{*}\right)}\left[\boldsymbol{\Theta}_{b^{*}}^{a^{*}}\right]_{\left(n_{r}^{*}, n_{r}^{*}\right)}\right\} .
\end{aligned}
$$

its coding and modulation mode according to the distance between the desired communicating aircraft. Based on our asymptotic closed-form theoretical analysis, we have explicitly derived the set of distance-thresholds for the proposed ACM design and have provided a theoretical upper bound of the achievable spectral efficiency and throughput, which has considered the impact of realistic channel estimation error and of co-channel interference. Our extensive simulation results have validated our design and theoretical analysis. This study therefore has provided a practical high-data-rate and highspectral-efficiency solution for supporting the future Internet above the clouds.

\section{APPENDIX}

First, we have the following lemma.

Lemma 1: Let $\boldsymbol{A} \in \mathbb{C}^{N \times N}$ and $\boldsymbol{x} \sim \mathcal{C N}\left(\frac{1}{\sqrt{N}} \boldsymbol{m}, \frac{1}{N} \mathbf{\Upsilon}\right)$, where $\frac{1}{\sqrt{N}} \boldsymbol{m} \in \mathbb{C}^{N \times 1}$ and $\frac{1}{N} \Upsilon \in \mathbb{C}^{N \times N}$ are the mean vector and the covariance matrix of $\boldsymbol{x}$, respectively. Assume that $\boldsymbol{A}$ has a uniformly bounded spectral norm with respect to $N$ and $\boldsymbol{x}$ is independent of $\boldsymbol{A}$. Then we have

$$
\lim _{N \rightarrow \infty} \boldsymbol{x}^{\mathrm{H}} \boldsymbol{A} \boldsymbol{x}=\operatorname{Tr}\left\{\left(\frac{1}{N} \boldsymbol{M}+\frac{1}{N} \boldsymbol{\Upsilon}\right) \boldsymbol{A}\right\},
$$

where $\boldsymbol{M}=\boldsymbol{m} \boldsymbol{m}^{\mathrm{H}}$.

Proof: Let $\boldsymbol{y}=\sqrt{N} \boldsymbol{x}-\boldsymbol{m}$. As $\boldsymbol{x} \sim \mathcal{C N}\left(\frac{1}{\sqrt{N}} \boldsymbol{m}, \frac{1}{N} \boldsymbol{\Upsilon}\right)$, we have $\boldsymbol{y} \sim \mathcal{C N}(\mathbf{0}, \mathbf{\Upsilon})$. Furthermore, we have

$$
\begin{gathered}
\boldsymbol{x}^{\mathrm{H}} \boldsymbol{A} \boldsymbol{x}=\left(\frac{1}{\sqrt{N}} \boldsymbol{m}+\frac{1}{\sqrt{N}} \boldsymbol{y}\right)^{\mathrm{H}} \boldsymbol{A}\left(\frac{1}{\sqrt{N}} \boldsymbol{m}+\frac{1}{\sqrt{N}} \boldsymbol{y}\right) \\
=\frac{1}{N} \boldsymbol{m}^{\mathrm{H}} \boldsymbol{A} \boldsymbol{m}+\frac{1}{N} \boldsymbol{y}^{\mathrm{H}} \boldsymbol{A} \boldsymbol{y}+\frac{1}{N} \boldsymbol{m}^{\mathrm{H}} \boldsymbol{A} \boldsymbol{y}+\frac{1}{N} \boldsymbol{y}^{\mathrm{H}} \boldsymbol{A} \boldsymbol{m} .
\end{gathered}
$$

Since $\boldsymbol{y} \sim \mathcal{C N}(\mathbf{0}, \boldsymbol{\Upsilon})$ and $\boldsymbol{y}$ does not depend on $\boldsymbol{m}$, according to Lemma 1 of [43], we have

$$
\begin{aligned}
& \lim _{N \rightarrow \infty} \frac{\boldsymbol{m}^{\mathrm{H}} \boldsymbol{A} \boldsymbol{y}}{N}=0, \\
& \lim _{N \rightarrow \infty} \frac{\boldsymbol{y}^{\mathrm{H}} \boldsymbol{A} \boldsymbol{m}}{N}=0 .
\end{aligned}
$$

Furthermore, according to the trace lemma of [44], we have

$$
\lim _{N \rightarrow \infty}\left(\frac{1}{\sqrt{N}} \boldsymbol{y}^{\mathrm{H}}\right) \boldsymbol{A}\left(\frac{1}{\sqrt{N}} \boldsymbol{y}\right)=\operatorname{Tr}\left\{\frac{1}{N} \boldsymbol{\Upsilon} \boldsymbol{A}\right\} .
$$

Substituting (41) to (43) into (40) leads to (39).
Recalling the distribution (20), we have

$$
\mathcal{E}\left\{\left[\widehat{\boldsymbol{H}}_{b^{*}}^{a^{*}}\right]_{\left[n_{r}^{*}:\right]}^{\mathrm{H}}\left[\widehat{\boldsymbol{H}}_{b^{*}}^{a^{*}}\right]_{\left[n_{r}^{*}:\right]}\right\}=\left[\boldsymbol{\Theta}_{b^{*}}^{a^{*}}\right]_{\left(n_{r}^{*}, n_{r}^{*}\right)} .
$$

Upon setting $\boldsymbol{x}=\left[\widehat{\boldsymbol{H}}_{b^{*}}^{a^{*}}\right]_{\left[n_{r}^{*}:\right]}$ in conjunction with $\boldsymbol{A}=\boldsymbol{I}_{N_{t}}$ in Lemma 1, we have

$$
\lim _{N_{t} \rightarrow \infty}\left[\widehat{\boldsymbol{H}}_{b^{*}}^{a^{*}}\right]_{\left[n_{r}^{*}:\right]}\left[\widehat{\boldsymbol{H}}_{b^{*}}^{a^{*}}\right]_{\left[n_{r}^{*}:\right]}^{\mathrm{H}}=\operatorname{Tr}\left\{\left[\boldsymbol{\Theta}_{b^{*}}^{a^{*}}\right]_{\left(n_{r}^{*}, n_{r}^{*}\right)}\right\} \text {. }
$$

Hence, for a large $N_{t}$, which is the case considered in this paper, we have

$$
\left[\widehat{\boldsymbol{H}}_{b^{*}}^{a^{*}}\right]_{\left[n_{r}^{*}:\right]}\left[\widehat{\boldsymbol{H}}_{b^{*}}^{a^{*}}\right]_{\left[n_{r}^{*}:\right]}^{\mathrm{H}} \approx \operatorname{Tr}\left\{\left[\boldsymbol{\Theta}_{b^{*}}^{a^{*}}\right]_{\left(n_{r}^{*}, n_{r}^{*}\right)}\right\} .
$$

In addition, according to the distribution of $\left[\widetilde{\boldsymbol{H}}_{b^{*}}^{a^{*}}\right]_{\left[n_{r}^{*}:\right]}$ given in (25), we have

$$
\mathcal{E}\left\{\left[\widetilde{\boldsymbol{H}}_{b^{*}}^{a^{*}}\right]_{\left[n_{r}^{*}:\right]}^{\mathrm{H}}\left[\widetilde{\boldsymbol{H}}_{b^{*}}^{a^{*}}\right]_{\left[n_{r}^{*}:\right]}\right\}=\left[\boldsymbol{\Xi}_{b^{*}}^{a^{*}}\right]_{\left(n_{r}^{*}, n_{r}^{*}\right)} .
$$

With the aid of (44) and (46) as well as (47), we can readily derive (30), as shown in (48) at the top of this page.

\section{REFERENCES}

[1] A. Jahn, et al., "Evolution of aeronautical communications for personal and multimedia services," IEEE Commun. Mag., vol. 41, no. 7, pp. 3643, Jul. 2003.

[2] Q. Vey, A. Pirovano, J. Radzik, and F. Garcia, "Aeronautical ad hoc network for civil aviation," in Proc. 6th Int. Workshop, Nets4Cars/Nets4Trains/Nets4Aircraft 2014 (Offenburg, Germany), May 6-7, 2014, pp. 81-93.

[3] M. Schnell, U. Epple, D. Shutin, and N. Schneckenburger, "LDACS: future aeronautical communications for air-traffic management," IEEE Commun. Mag., vol. 52, no. 5, pp. 104-110, May 2014.

[4] R. Jain, F. Templin, and K.-S. Yin, "Analysis of L-band digital aeronautical communication systems: L-DACS1 and L-DACS2," in Proc. 2011 IEEE Aerospace Conf. (Big Sky, Montana), Mar. 5-12, 2011, pp. 1-10.

[5] J. M. Budinger and E. Hall, "Aeronautical mobile airport communications system (AeroMACS)," NASA/TM-2011-217236, NASA Glenn Research Center, Oct. 2011.

[6] G. Bartoli, R. Fantacci, and D. Marabissi, "AeroMACS: a new perspective for mobile airport communications and services," IEEE Wireless Commun., vol. 20, no. 6, pp. 44-50, Dec. 2013.

[7] T. Gräupl, M. Ehammer, and S. Zwettler, "L-DACS1 air-to-air data-link protocol design and performance," in Proc. ICNS 2011 (Herndon, VA), May 10-12, 2011, pp. 1-10.

[8] B. Haind, "An independent technology assessment for a future aeronautical communication system based on potential systems like B-VHF,' in Proc. DASC 2007 (Dallas, TX), Oct. 21-25, 2007, pp. 4.D.6-1-4.D.6-12.

[9] A. J. Goldsmith and S.-G. Chua, "Adaptive coded modulation for fading channels," IEEE Trans. Commun., vol. 46, no. 5, pp. 595-602, May 1998. 
[10] L. Hanzo, C. H. Wong, and M. S. Yee, Adaptive Wireless Transceivers: Turbo-Coded, Turbo-Equalised and Space-Time Coded TDMA, CDMA, MC-CDMA and OFDM Systems. John Wiley: New York, USA, 2002.

[11] P. H. Tan, Y. Wu, and S. Sun, "Link adaptation based on adaptive modulation and coding for multiple-antenna OFDM system," IEEE $J$. Sel. Areas Commun., vol. 26, no. 8, pp. 1599-1606, Oct. 2008.

[12] E. Dahlman, S. Parkvall, and J. Skold, 4G: LTE/LTE-advanced for Mobile Broadband. Academic Press, 2013.

[13] J. Meng and E.-H. Yang, "Constellation and rate selection in adaptive modulation and coding based on finite blocklength analysis and its application to LTE," IEEE Trans. Wireless Commun., vol. 13, no. 10, pp. 5496-5508, Oct. 2014.

[14] E. Alberty, S. Defever, C. Moreau, R. De Gaudenzi, A. Ginesi, R. Rinaldo, G. Gallinaro, and A. Vernucci, "Adaptive coding and modulation for the DVB-S2 standard interactive applications: capacity assessment and key system issues," IEEE Wireless Commun., vol. 14, no. 4, pp. 6169, Aug. 2007.

[15] S. Zhou and G. B. Giannakis, "Adaptive modulation for multiantenna transmissions with channel mean feedback," IEEE Trans. Wireless Commun., vol. 3, no. 5, pp. 1626-1636, Sep. 2004.

[16] S. Zhou and G. B. Giannakis, "How accurate channel prediction needs to be for transmit-beamforming with adaptive modulation over Rayleigh MIMO channels?" IEEE Trans. Wireless Commun., vol. 3, no. 4, pp. 1285-1294, Jul. 2004

[17] M. Taki, M. Rezaee, and M. Guillaud, "Adaptive modulation and coding for interference alignment with imperfect CSIT," IEEE Trans. Wireless Commun., vol. 13, no. 9, pp. 5264-5273, Sep. 2014.

[18] L. Hanzo, S. X. Ng, T. Keller, W. Webb, Quadrature Amplitude Modulation: From Basics to Adaptive Trellis-Coded, Turbo-Equalised and Space-Time Coded OFDM, CDMA and MC-CDMA Systems (Second Edition). Wiley-IEEE Press: New York, NY, USA, 2004.

[19] E. Haas, "Aeronautical channel modeling," IEEE Trans. Vehicular Technology, vol. 51, no. 2, pp. 254-264, Mar. 2002.

[20] S. Gligorevic, "Airport surface propagation channel in the C-Band: measurements and modeling," IEEE Trans. Antennas and Propagation, vol. 61, no. 9, pp. 4792-4802, Sep. 2013.

[21] C. Zhang, K. Pang, and L. Ma, "Interpolated airborne MIMO antenna array," IEEE Antennas and Wireless Propagation Let., vol. 14, pp. 72 $75,2015$.

[22] J. Zhang, T. Chen, S. Zhong, W. Zhang, X. Zuo, J. Darlington, R. G. Maunder, and L. Hanzo, "A survey of aeronautical ad-hoc networking," submitted to IEEE Commun. Surveys \& Tutorials, 2017.

[23] X. Gao, Z. Shen, and C. Hua, "Conformal VHF log-periodic balloon antenna," IEEE Trans. Antennas and Propagation, vol. 63, no. 6, pp. 2756-2761, Jun. 2015.

[24] DO-242A, "Minimum aviation system performance standards for automatic dependent surveillance-broadcast (ADS-B)," RTCA, Jun. 25, 2002.

[25] J. D. Parsons, The Mobile Radio Propagation Channel (2nd Edition). Wiley, 2000

[26] K. Kim, J. Lee, and H. Liu, "Spatial-correlation-based antenna grouping for MIMO systems," IEEE Trans. Vehicular Technology, vol. 59, no. 6, pp. 2898-2905, Jul. 2010.

[27] J. Zhang, S. Chen, X. Mu, and L. Hanzo, "Joint channel estimation and multiuser detection for SDMA/OFDM based on dual repeated weighted boosting search," IEEE Trans. Vehicular Technology, vol. 60, no. 7, pp. 3265-3275, Sep. 2011.

[28] R. E. Sheriff and Y. F. Hu, Mobile Satellite Communication Networks. John Wiley \& Sons, 2003.

[29] S. M. Kay, Fundamentals of Statistical Signal Processing: Estimation Theory. Prentice-Hall: Upper Saddle River, 2003.

[30] A. B. Gershman, N. D. Sidiropoulos, S. Shahbazpanahi, M. Bengtsson, and B. Ottersten, "Convex optimization-based beamforming: From receive to transmit and network designs," IEEE Signal Process. Mag., vol. 27, no. 3, pp. 62-75, May 2010.

[31] R. G. Lorenz and S. P. Boyd, "Robust minimum variance beamforming," IEEE Trans. Signal Process., vol. 53, no. 5, pp. 1684-1696, May 2005.

[32] W. Yao, S. Chen, S. Tan, and L. Hanzo, "Minimum bit error rate multiuser transmission designs using particle swarm optimisation," IEEE Trans. Wireless Commun., vol. 8, no. 10, pp. 5012-5017, Oct. 2009.

[33] B. R. Vojčić and W. M. Jang, "Transmitter precoding in synchronous multiuser communications," IEEE Trans. Commun., vol. 46, no. 10, pp. 1346-1355, Oct. 1998.

[34] J. Hoydis, S. Ten Brink, and M. Debbah, "Massive MIMO in the UL/DL of cellular networks: How many antennas do we need?" IEEE J. Sel. Areas Commun., vol. 31, no. 2, pp. 160-171, Feb. 2013.
[35] E. Sakhaee and A. Jamalipour, "The global in-flight Internet," IEEE $J$. Sel. Areas Commun., vol. 24, no. 9, pp. 1748-1757, Sep. 2006.

[36] S. Gong, C. Xing, S. Chen, and Z. Fei, "Secure communications for dual-polarized MIMO systems," IEEE Trans. Signal Process., vol. 65, no. 16, pp. 4177-4192, Aug. 2017.

[37] S. Gong, C. Xing, S. Chen, N. Yang, and Y. Zhou, "Robust energyefficient precoding optimization for dual-polarized multiuser MIMO downlink," to be presented at Proc. ICCC 2017 (Chengdu, China), Dec. 13-16, 2017, pp. 1-5.

[38] Comtech EF Data, "VersaFEC," http://www.comtechefdata.com/ technologies/fec/versafec, Accessed on February 3rd, 2016, [[Online]. Available]

[39] H. A. Ngo and L. Hanzo, "Hybrid automatic-repeat-request systems for cooperative wireless communications," IEEE Commun. Surveys \& Tutorials, vol. 16, no. 1, pp. 25-45, 2014.

[40] H. Chen, R. G. Maunder, and L. Hanzo, "A survey and tutorial on lowcomplexity turbo coding techniques and a holistic hybrid ARQ design example," IEEE Commun. Surveys \& Tutorials, vol. 15, no. 4, pp. 15461566,2013

[41] C. Martin and B. Ottersten, "Asymptotic eigenvalue distributions and capacity for MIMO channels under correlated fading," IEEE Trans. Wireless Commun., vol. 3, no. 4, pp. 1350-1359, Jul. 2004.

[42] B. Lee, J. Choi, J.-Y. Seol, D. J. Love, and B. Shim, "Antenna grouping based feedback compression for FDD-based massive MIMO systems," IEEE Trans. Commun., vol. 63, no. 9, pp. 3261-3274, Sep. 2015.

[43] F. Fernandes, A. Ashikhmin, and T. L. Marzetta, "Inter-cell interference in noncooperative TDD large scale antenna systems," IEEE J. Sel. Areas Commun., vol. 31, no. 2, pp. 192-201, Feb. 2013.

[44] J. Hoydis, "Random matrix theory for advanced communication systems," Ph.D. dissertation, Supélec, 2012.

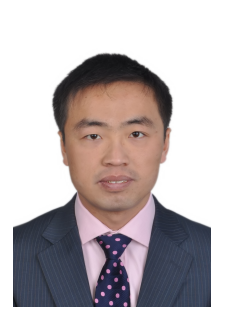

Jiankang Zhang (S'08-M'12) received the B.Sc. degree in Mathematics and Applied Mathematics from Beijing University of Posts and Telecommunications in 2006, and the Ph.D. degree in Communication and Information Systems from Zhengzhou University in 2012. Since then, he has been a lecturer in School of Information Engineering, Zhengzhou University. From 2009 to 2011, Dr Zhang was a visiting researcher in the School of Electronics and Computer Science, the University of Southampton, UK. From 2013 to 2014, Dr Zhang was a postdoctoral researcher in the McGill University, Canada. From 2014, he has been a research fellow in the University of Southampton, UK. His research interests are in the areas of wireless communications and signal processing, aircraft communication and wirelines communication.

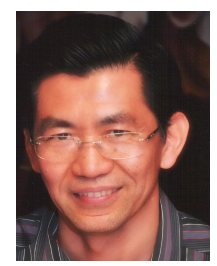

Sheng Chen (M'90-SM'97-F'08) received his BEng degree from the East China Petroleum Institute, Dongying, China, in 1982, and his $\mathrm{PhD}$ degree from the City University, London, in 1986, both in control engineering. In 2005, he was awarded the higher doctoral degree, Doctor of Sciences (DSc), from the University of Southampton, Southampton, UK. From 1986 to 1999, He held research and academic appointments at the Universities of Sheffield, Edinburgh and Portsmouth, all in UK. Since 1999, he has been with the School of Electronics and Computer Science, the University of Southampton, UK, where he holds the post of Professor in Intelligent Systems and Signal Processing. Dr Chen's research interests include adaptive signal processing, wireless communications, modelling and identification of nonlinear systems, neural network and machine learning, intelligent control system design, evolutionary computation methods and optimisation. He has published over 550 research papers. Dr. Chen is a Fellow of the United Kingdom Royal Academy of Engineering, a Fellow of IET, a Distinguished Adjunct Professor at King Abdulaziz University, Jeddah, Saudi Arabia, and an ISI highly cited researcher in engineering (March 2004). 


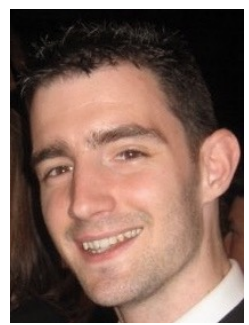

Robert Maunder (SM'12) received a first class honours BEng degree in Electronic Engineering in July 2003 and the $\mathrm{PhD}$ degree in Telecommunications in December 2007, from the University of Southampton. He began a lectureship in November 2007 with the School of Electronics and Computer Science, the University of Southampton, UK, and was promoted to Associate Professor in March 2013 and to Professor in August 2017. Professor Maunder's research interests include joint source/channel coding and the holistic design of algorithms and hardware implementations for wireless communications. He has published a number of IEEE papers in these areas. He is the founder and CTO of AccelerComm Ltd, which is commercialising his research as soft-IP. Dr Maunder is a Chartered Engineer and a Fellow of the IET.

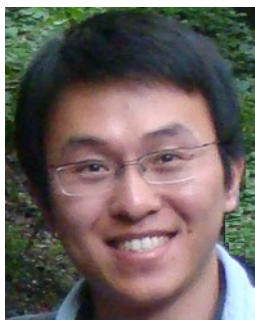

Rong Zhang (M'09-SM'16) received his $\mathrm{PhD}$ in wireless communications from the University of Southampton (UoS) in 2009, where he was a research assistant during that period with the Mobile Virtual Centre of Excellence, one of UK's largest industrial-academic partnership in ICT. During his post-doctoral period with the School of Electronics and Computer Science, the UoS, he contributed, as a UoS lead researcher, to a number of international projects. After that, he took his industrial consulting leave for Huawei EU R\&D as a System Algorithms Expert. Currently, he is an assistant professor in Southampton Wireless Group, the UoS. Dr Zhang has a total of 80+ IEEE/OSA publications, including 55+ journals $(20+$ of which as the first author). Owing to his outstanding academic achievements, he is the recipient of the prestigious Dean's Publication Award, Faculty of Physical Sciences and Engineering, the UoS. He is also the recipient of the prestigious RAEng industrial fellowship. He regularly serves as reviewer for IEEE/OSA journals and funding bodies and has been several times as TPC member/invited session chair of major conferences. He is a RAEng industrial fellow and a member of the OSA.

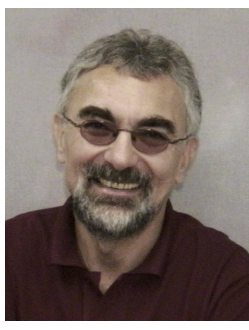

Lajos Hanzo FREng, FIEEE, FIET, Fellow of EURASIP, DSc, received his degree in electronics in 1976 and his doctorate in 1983. In 2009 he was awarded an honorary doctorate by the Technical University of Budapest and in 2015 by the University of Edinburgh. In 2016 he was admitted to the Hungarian Academy of Science. During his 40-year career in telecommunications he has held various research and academic posts in Hungary, Germany and the UK. Since 1986 he has been with the School of Electronics and Computer Science, University of Southampton, UK, where he holds the chair in telecommunications. He has successfully supervised $111 \mathrm{PhD}$ students, co-authored 18 John Wiley/IEEE Press books on mobile radio communications totalling in excess of 10,000 pages, published 1701 research contributions at IEEE Xplore, acted both as TPC and General Chair of IEEE conferences, presented keynote lectures and has been awarded a number of distinctions. Currently he is directing an academic research team, working on a range of research projects in the field of wireless multimedia communications sponsored by industry, the Engineering and Physical Sciences Research Council (EPSRC) UK, the European Research Council's Advanced Fellow Grant and the Royal Society's Wolfson Research Merit Award. He is an enthusiastic supporter of industrial and academic liaison and he offers a range of industrial courses. He is also a Governor of the IEEE VTS. During 2008-2012 he was the Editor-in-Chief of the IEEE Press and a Chaired Professor also at Tsinghua University, Beijing. For further information on research in progress and associated publications please refer to http://wwwmobile.ecs.soton.ac.uk. Professor Hanzo has 30,000+ Google Scholar citations and an H-index of 70. 